\title{
Developing convective flow in a square channel partially filled with a high porosity metal foam and rotating in a parallel-mode
}

DOI:

10.1016/j.ijheatmasstransfer.2015.06.080

\section{Document Version}

Submitted manuscript

Link to publication record in Manchester Research Explorer

Citation for published version (APA):

Alhusseny, A., Turan, A., \& Nasser, A. (2015). Developing convective flow in a square channel partially filled with a high porosity metal foam and rotating in a parallel-mode. International Journal of Heat and Mass Transfer, 90, 578590. https://doi.org/10.1016/j.ijheatmasstransfer.2015.06.080

\section{Published in:}

International Journal of Heat and Mass Transfer

\section{Citing this paper}

Please note that where the full-text provided on Manchester Research Explorer is the Author Accepted Manuscript or Proof version this may differ from the final Published version. If citing, it is advised that you check and use the publisher's definitive version.

\section{General rights}

Copyright and moral rights for the publications made accessible in the Research Explorer are retained by the authors and/or other copyright owners and it is a condition of accessing publications that users recognise and abide by the legal requirements associated with these rights.

\section{Takedown policy}

If you believe that this document breaches copyright please refer to the University of Manchester's Takedown Procedures [http://man.ac.uk/04Y6Bo] or contact uml.scholarlycommunications@manchester.ac.uk providing relevant details, so we can investigate your claim.

\section{OPEN ACCESS}


Elsevier Editorial System(tm) for International Journal of Heat and Mass Transfer Manuscript Draft

Manuscript Number: HMT-D-15-00716R1

Title: Developing Convective Flow in a Square Channel Partially Filled with a High Porosity Metal Foam and Rotating in a Parallel-Mode

Article Type: Full Length Article

Keywords: High Porosity Foam; Rotation; Channel; Thermal Dispersion; Partial Occupation; Interface

Corresponding Author: Mr. Ahmed Niameh Mehdy Alhusseny,

Corresponding Author's Institution: University of Manchester

First Author: Ahmed Niameh Mehdy Alhusseny

Order of Authors: Ahmed Niameh Mehdy Alhusseny; Ali Turan, Professor ; Adel Nasser, Dr

Abstract: The development of three-dimensional heat transfer and fluid flow in a square channel rotating in a parallel-mode has been investigated numerically. The duct is partially occupied by a foam material of high porosity $\varepsilon \geq 0.89$ and subjected to a uniform wall heat flux. In regards to the influence of rotation, both the centrifugal buoyancy and Coriolis forces are considered in the current study. The generalised model is used to mathematically simulate the momentum equations employing the Boussinesq approximation for the density variation. Moreover, thermal dispersion has been taken into account with considering that fluid and solid phases are in a local thermal non-equilibrium. The governing equations are discretised according to the finite volume method with employing a hybrid differencing scheme. Computations are performed for a wide range of parameters including the hollow ratio $(0 \leq S \leq 1)$, foam porosity $(0.89 \leq \varepsilon \leq 0.97)$, pore density ( $5 \mathrm{PPI} \leq \omega \leq 40 \mathrm{PPI})$, solid to fluid thermal conductivity ratio $(250 \leq \kappa \leq 4000)$, Reynolds number $(250 \leq \mathrm{Re} \leq 2000)$, and rotation number $(0 \leq \mathrm{R} 0 \leq 1)$, while the values of characteristic temperature difference and Prandtl numbers are maintained constant at $\Delta \mathrm{Tc}=1000^{\circ} \mathrm{C}$ and $\mathrm{Pr}=0.7$, respectively. Results reveal that flow resistance and heat transport are augmented with either decreasing the hollow ratio and foam porosity or increasing Reynolds and rotation numbers, while two contradictory trends are found for the impact of increasing pore density on heat transfer; either enhancing or suppressing depending on the size of hollow zone. In addition, both rotation and thermal dispersion have dominant roles in enhancing heat transfer at the higher levels of porosity or the lower values of conductivity ratios. However, these roles are reduced gradually with decreasing the foam porosity or increasing thermal conductivity ratio, but do not completely vanish. Eventually, the worth of using high porosity fibrous media in enhancing the heat transported through rotating channels has been inspected. An overall enhancement parameter is compared for the current study with a previous work regarding turbulent flow in a rotating clear channel, where it has been confirmed that the current proposal is practically justified and efficient. 


\author{
Ahmed Alhusseny \\ A5, George Begg Building, Sackville Street \\ Manchester, M60 1QD \\ (+44) 7876340153 \\ ahmed.alhusseny@ postgrad.manchester.ac.uk
}

\title{
International Journal of Heat and Mass Transfer
}

The current research presents a numerical simulation of the three-dimensional fluid flow and heat transfer in a square channel rotating around a parallel axis. The duct is partially occupied by metal foam of high porosity $(\varepsilon \geq 0.89)$ and subjected to a uniform wall heat flux. Both the centrifugal buoyancy effect and Coriolis forces are considered regarding to the rotation effect in the current study.

The generalised model is used to mathematically simulate the momentum equations employing the Boussinesq approximation for the density variation. Moreover, thermal dispersion has been taken into account with considering that fluid and solid phases are in a local thermal non-equilibrium. An iterative method has been used to solve the governing equations, which have been discretised according to the finite volume method. Computations are performed for a wide range of parameters including the hollow ratio, foam porosity, pore density, solid to fluid-phase thermal conductivity ratio, Reynolds number, and rotation number with keeping the characteristic temperature difference and Prandtl number at constant values. Detailed analysis is presented for heat transfer improvement by means of changing the rotation rate or the porous medium characteristics such as the hollow ratio, medium porosity, pore density, and the solid to fluid-phase thermal conductivity ratio.

It is anticipated that a fair evaluation will be carried out by the reviewers and valuable observations/recommendations will be provided for the improvement of the present or future works.

\section{Sincerely,}

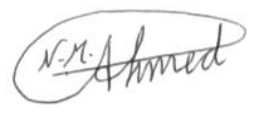

Signature

Ahmed Alhusseny 


\section{Detailed Response to Reviewers}

\begin{tabular}{|c|c|}
\hline The Comments of Reviewer 1 & Response to Reviewer 1 \\
\hline $\begin{array}{l}\text { The lack of this information is } \\
\text { evident in the } 3 \text { stated highlights } \\
\text { which are merely stating what the } \\
\text { authors have done. On reading } \\
\text { these highlights then it does not } \\
\text { encourage the reader to read the } \\
\text { complete manuscript. } \\
\text { My recommendation is that the } \\
\text { authors substantially reduce the } \\
\text { length of the manuscript and make } \\
\text { it much more focused on the major } \\
\text { conclusions. Further, the study of a } \\
\text { more physical set up would } \\
\text { substantially enhance the } \\
\text { contribution. }\end{array}$ & $\begin{array}{l}\text { The section of research highlights has been rewritten and modified to be } \\
\text { more detailed and informative by including the most important findings } \\
\text { obtained in the current study. Moreover, the manuscript was made more } \\
\text { concise by reducing the length of some of its paragraphs. }\end{array}$ \\
\hline $\begin{array}{l}\text { The words "in parallel mode" needs } \\
\text { to made clearer- parallel to what? }\end{array}$ & $\begin{array}{l}\text { The expression "rotation in parallel mode" refers to flow in passages } \\
\text { parallel to the axis of rotation, or in other words, channels rotating about } \\
\text { an axis parallel to the direction of the main stream. This term has been } \\
\text { more clarified in the first paragraph of each one of section-1 } \\
\text { (Introduction) and section-2 (Mathematical formulation). }\end{array}$ \\
\hline $\begin{array}{l}\text { Why is a uniform wall heat flux } \\
\text { taken? Why not take a more } \\
\text { physical boundary condition? At the } \\
\text { moment, this manuscript is of } \\
\text { mainly mathematical interest. }\end{array}$ & $\begin{array}{l}\text { In most of the former experimental or numerical works, which tackled the } \\
\text { problem of developing convective flow in channels rotating about a } \\
\text { parallel axis, a uniform wall heat flux was applied as a thermal boundary } \\
\text { condition at the channel walls. To name a few: } \\
\text { 1- W.D. Morris, Heat transfer and fluid flow in rotating coolant channels, } \\
\text { Research Studies Pr./John Wiley and Sons, Chichester, } 1981 \text {. } \\
\text { 2- E. Levy, S. Neti, G. Brown, F. Bayat, V. Kadambi, Laminar heat transfer } \\
\text { and pressure drop in a rectangular duct rotating about a parallel axis, } \\
\text { J. Heat Transfer } 108 \text { (2) (1986) 350-356. } \\
\text { 3- A.K. Sleiti, J.S. Kapat, Heat transfer in channels in parallel-mode } \\
\text { rotation at high rotation numbers, Journal of Thermophysics and Heat } \\
\text { Transfer } 20 \text { (4) (2006) 748-753. } \\
\text { Therefore, a uniform wall heat flux has been considered as a thermal } \\
\text { boundary condition at the channel walls in the current study. }\end{array}$ \\
\hline $\begin{array}{l}\text { The authors keep stating THE } \\
\text { hybrid method but it is A hybrid } \\
\text { method. }\end{array}$ & $\begin{array}{l}\text { The expression "the hybrid method" has been corrected into "a hybrid } \\
\text { method" in both the "Abstract" and "Solution procedure" sections. }\end{array}$ \\
\hline $\begin{array}{l}\text { The authors claim to have studied a } \\
\text { wide range of parameters - this is } \\
\text { not true as only one value of some } \\
\text { of the parameters has been studied. }\end{array}$ & $\begin{array}{l}\text { Multiple parameters were examined in the current study, and their impact } \\
\text { was presented for a wide range of each one of them. For example, the } \\
\text { hollow ratio }(0 \leq S \leq 1) \text { as shown in Figs. }(3,4,5,6,7,10,12,13,14,15) \text {, } \\
\text { medium porosity has been varied from } 0.89 \text { to } 0.97 \text { as shown in Figs. }(6,9 \text {, } \\
10,12,13,14) \text {, pore density ( } 5 \mathrm{PPI} \leq \omega \leq 40 \mathrm{PPI}) \text { as shown in Figs. }(6,7,8,9 \text {, } \\
\text { 10), solid to fluid-phase thermal conductivity ratio }(250 \leq \mathrm{K} \leq 4000) \text { as shown } \\
\text { in Fig.(14), rotation number }(0 \leq \mathrm{Ro} \leq 1.0) \text { as shown in Figs. }(11,12,13) \text {, and } \\
\text { Reynolds number ( } 250 \leq \mathrm{Re} \leq 2000) \text { as shown in Fig.(15.a). We know that in } \\
\text { some cases an only one value of a certain parameter was considered, but } \\
\text { this happened while changing other parameters to investigate their } \\
\text { impact because it is quite difficult to change multiple parameters } \\
\text { simultaneously and present their impact together in a single figure. }\end{array}$ \\
\hline
\end{tabular}


I do not understand how the last word in the abstract, namely, 'efficient' has been justified.
It means that using metal foams in filling cooling passages, which rotate about a parallel axis, offers a high heat transfer rate compared to the pressure drop produced especially at the extreme levels of rotation unlike using turbulent flow in clear channels. In addition, it can dissipate the heat accumulated at channel walls in levels comparable to those obtained by passing a turbulent flow through clear channels. This outcome is detailed and clarified in the section-4.5 (Overall Enhancement) of the manuscript.

Not all symbols are defined, e.g. gamma, and symbols should be listed alphabetically.

Why study a rectangular cavity? Is this a physical situation? all symbol used are defined clearly and listed alphabetically.

The studied problem is convective flow in a square channel, which is one of the passages forms used in cooling the rotor windings of electrical
The Nomenclature section was rechecked and modified to make sure that generators as stated by "A.K. Sleiti, J.S. Kapat, Heat transfer in channels in parallel-mode rotation at high rotation numbers, Journal of Thermophysics and Heat Transfer 20 (4) (2006) 748-753.".

The Reynolds number investigated is 2,000 and the flow is assumed range of Reynolds numbers should be considered.

The validation of the use of the velocity inlet boundary condition requires a comment.

The thermal boundary conditions on $Y=0,1$ and $Z=0,1$ needs explanation as the normal direction is different on the faces at 0 and 1.

In table 1 , surely results are not accurate to 6 decimal places.

A range of Reynolds number $(250 \leq \operatorname{Re} \leq 2000)$ has been considered and its impact on the heat transported and overall performance obtained has been illustrated in Fig.(15.a) and discussed in section-4.5 (Overall enhancement).

As the flow is assumed hydrodynamically and thermally developing, velocity profile at the inlet needs to be uniform in order to properly establish the process of boundary layer development. This is explained in the paragraph located just before Eq.(19) in the manuscript.

The thermal boundary conditions at $Y=0,1$ and $Z=0,1$ has been rewritten and explained at each one of the faces located at 0 and 1 , as shown in Eq.(19).

The six decimal places were used to check the error at each iteration as well as the data files used to plot the results obtained, while there was no laminar - this needs explanation. A need to mention all the six decimal places in Table 1 because the data presented there are all of order one or more in addition to that the accuracy of Nusselt number values in the above mentioned table is not noticeably improved by mentioning the remaining decimal points.

There are oscillations in some of the results, e.g. figure 5 . Is this due to the assumption of laminar flow, incorrectly dealing with the singularity at $\mathrm{X}=0$, physical, or something else? Please explain.

I presume that a constant mesh size is used in the $X$ direction. In practice a very small mesh is required near $\mathrm{X}=0$ and $\mathrm{as}$ increases a larger mesh can be applied.
The existence of these oscillations in Fig.(5) is due to the formation and vanishing of additional cross-sectional vortices along the channel, especially at the higher hollow ratios $S \geq 0.6$, where the centrifugal buoyancy becomes less dominant and Coriolis forces start to play a more obvious and influential role. This leads to break down the symmetry around the mid-vertical axis $Z=0.5$ and creates extra transverse vortices allow the boundary layer to be thinner and then becomes thicker again when these vortices vanish, and hence, the heat transfer level and pressure gradient oscillate as well.

The mesh has been already refined in the axial direction at the entrance region, where more grid points are clustered near the channel inlet according to the practice suggested by "K. Hoffmann and S. Chiang, Computational Fluid Dynamics, 4th ed. vol. 1. Engineering Education Science 1 , USA (2000), pp. 369" as:

$X_{i}=L_{X}\left\{(\beta+1)-(\beta-1)[(\beta+1) /(\beta-1)]^{\left(1-\xi_{i}\right)}\right\} /\left\{[(\beta+1) /(\beta-1)]^{\left(1-\xi_{i}\right)}+1\right\}$ Where $\boldsymbol{i}$ is the grid index in X-direction (i=1 to $\boldsymbol{n i}), \boldsymbol{L}$ is the channel length, $\boldsymbol{\beta}$ is the clustering parameter within the range of $\boldsymbol{I}$ to $\infty$. As the value of $\boldsymbol{\beta}$ 


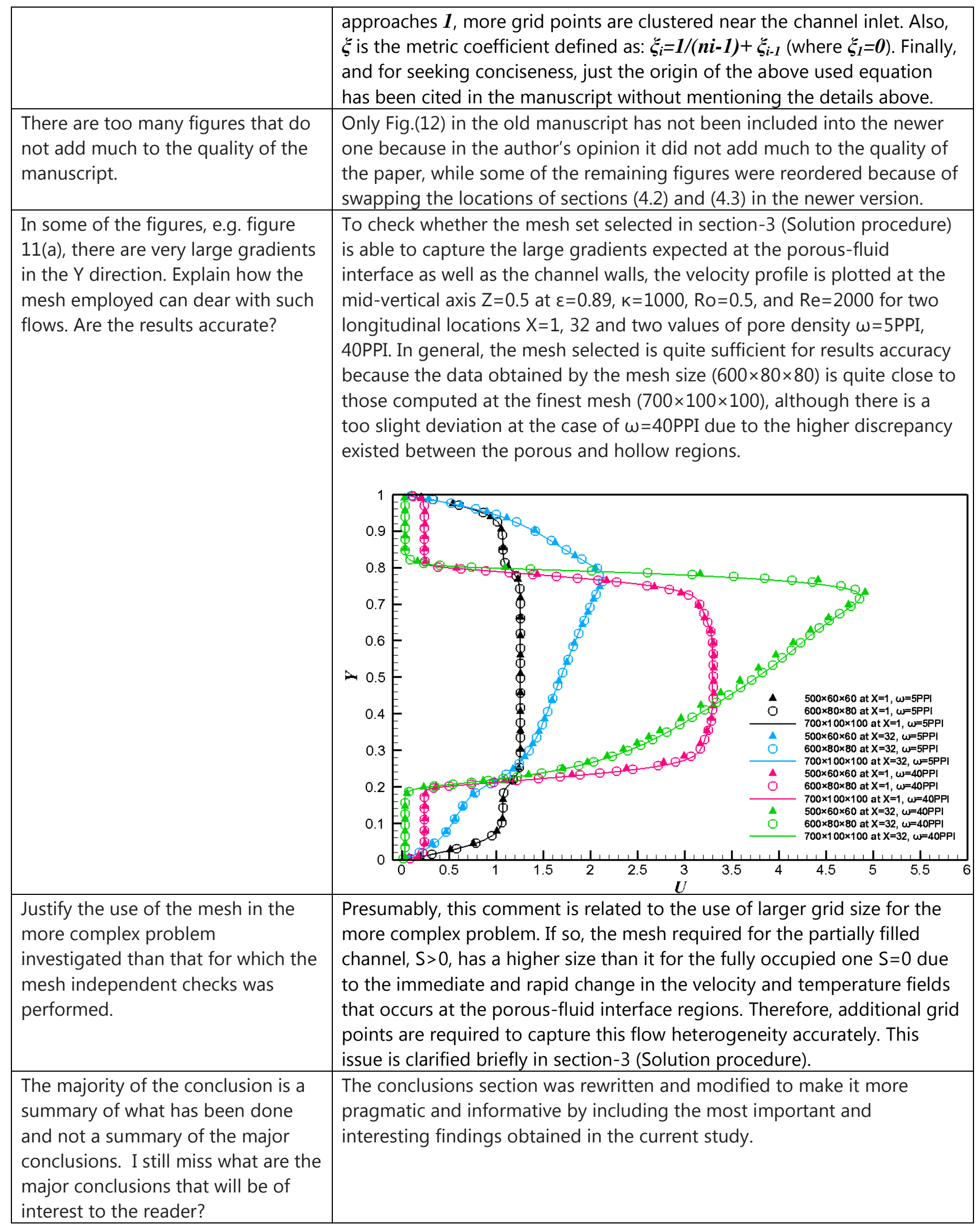




\begin{tabular}{|c|c|}
\hline The Comments of Reviewer 2 & Response to Reviewer 2 \\
\hline $\begin{array}{l}\text { The authors only referred to the } \\
\text { works with LTE assumption (page } \\
\text { 4). To complete the authors } \\
\text { statement, the authors can benefit } \\
\text { by taking into consideration the } \\
\text { following analytical/numerical } \\
\text { works which have fully utilized the } \\
\text { LTNE assumption. }\end{array}$ & $\begin{array}{l}\text { Some former works recommended by the reviewer have already been } \\
\text { cited in the manuscript such as: } \\
\text { 1- B. Alazmi, K. Vafai, Constant wall heat flux boundary conditions in } \\
\text { porous media under local thermal non-equilibrium conditions, Int. J. } \\
\text { Heat Mass Transfer } 45 \text { (2002) 3071-3087. } \\
\text { 2- S. Mahjoob, K. Vafai, A synthesis of fluid and thermal transport models } \\
\text { for metal foam heat exchangers, Int. J. Heat Mass Transfer } 51 \text { (2008) } \\
\text { 3701-3711. } \\
\text { While additional works suggested by the reviewer, where the utilization of } \\
\text { LTNE was taken into account, have been recently cited in the introduction } \\
\text { section. Those are: } \\
\text { 1- A. Amiri, K. Vafai, T.M. Kuzay, Effects of boundary conditions on non- } \\
\text { darcian heat transfer through porous media and experimental } \\
\text { comparisons, Numerical Heat Transfer, Part A (1995) 651-664. } \\
\text { 2- D-Y. Lee, K. Vafai, Analytical characterization and conceptual } \\
\text { assessment of solid and fluid temperature differentials in porous } \\
\text { media, Int. J. Heat Mass Transfer } 42 \text { (1999) } 423-435 \text {. } \\
\text { 3- S. Mahjoob, K. Vafai, Analytical characterization and production of an } \\
\text { isothermal surface for biological and electronic applications, J. Heat } \\
\text { Transfer } 131 \text { (2009) p. } 052604 \text {. } \\
\text { 4- S. Mahjoob, K. Vafai, Analysis of heat transfer in consecutive variable } \\
\text { cross-sectional domains: applications in biological media and thermal } \\
\text { management, J. Heat Transfer } 133 \text { (2011) p. 011006. }\end{array}$ \\
\hline $\begin{array}{l}\text { Please state more applications of } \\
\text { the research (rotational foam filled } \\
\text { channels). }\end{array}$ & $\begin{array}{l}\text { An additional application of rotational foam filled channels is utilizing the } \\
\text { metal foam to improve the internal cooling of turbine blades, as proposed } \\
\text { by "S.C. Tzeng, C.Y. Soong, S.C. Wong, Heat transfer in rotating channel } \\
\text { with open cell porous aluminum foam, Int. Commun. Heat Mass Transfer } \\
31 \text { (2) (2004) 261-272.". }\end{array}$ \\
\hline $\begin{array}{l}\text { Please double check to make sure } \\
\text { all symbols are properly typed and } \\
\text { converted to PDF format (please } \\
\text { check pages } 3,5 \text { ). }\end{array}$ & $\begin{array}{l}\text { All symbols were rechecked to make sure that they are properly typed and } \\
\text { converted to PDF format. }\end{array}$ \\
\hline $\begin{array}{l}\text { It is stated in Abstract and } \\
\text { conclusion that "flow resistance is } \\
\text { reduced with increasing the } \\
\text { porosity and hollow ratio or } \\
\text { decreasing pore density, while heat } \\
\text { transport is generally enhanced." } \\
\text { However, as the data indicate, the } \\
\text { heat transport is generally } \\
\text { decreased and not enhanced (page } \\
\text { 13). }\end{array}$ & $\begin{array}{l}\text { The mentioned statement has been corrected as "flow resistance and heat } \\
\text { transport are augmented with either decreasing the hollow ratio and foam } \\
\text { porosity or increasing Reynolds and rotation numbers, while two } \\
\text { contradictory trends are found for the impact of increasing pore density } \\
\text { on heat transfer; either enhancing or suppressing depending on the size } \\
\text { of hollow zone". }\end{array}$ \\
\hline $\begin{array}{l}\text { Please indicate all the units such as } \\
\text { in velocity contours in Figs. } 3,6 \text {. }\end{array}$ & $\begin{array}{l}\text { The values of velocity illustrated in Figs. }(3,6) \text { are all dimensionless. So, } \\
\text { there is no need to indicate their units. }\end{array}$ \\
\hline
\end{tabular}


Paper title:

\section{Developing Convective Flow in a Square Channel Partially Filled with a High Porosity Metal Foam and Rotating in a Parallel-Mode}

\section{First author details:}

Name: Ahmed Niameh Mehdy Alhusseny

Address: School of Mechanical, Aerospace, and Civil Engineering, the University of Manchester, P.O. Box 88, Manchester, M60 1QD, UK

Email: Ahmed.Alhusseny@ postgrad.manchester.ac.uk

Mobile: +44(0)7876340153

\section{Second author details:}

Name: Prof Ali Turan

Address: School of Mechanical, Aerospace, and Civil Engineering, the University of Manchester, P.O. Box 88, Manchester, M60 1QD, UK

Email: A.Turan@postgrad.manchester.ac.uk

\section{Third author details:}

Name: Dr Adel Nasser

Address: School of Mechanical, Aerospace, and Civil Engineering, the University of Manchester, P.O. Box 88, Manchester, M60 1QD, UK

Email: a.g.nasser@manchester.ac.uk 


\section{Research Highlights}

- Numerical simulation of flow and heat transfer development in a channel partially filled with high porosity metal foam and rotating about a parallel axis.

- Enlarging the hollow region up to a particular extent $S \approx 0.6$ leads to improving the overall performance $(\mathrm{Nu} / \Delta \mathrm{P})$ obtained although it reduces the heat transport.

- Despite the fact that reducing foam porosity causes higher pressure drop, it enhances heat transfer considerably, and hence, improves the overall performance significantly.

- Two contradictory trends are found for the impact of increasing pore density on heat transfer; either enhancing or suppressing depending on the size of hollow zone, while the pressure loss is considerably augmented, and hence, the overall performance deteriorates.

- Increasing rotation strength improves both the heat transport and overall performance although it causes higher pressure loss.

- Thermal dispersion plays an influential role in enhancing heat transfer especially at the higher values of foam porosity or the lower values of either the hollow ratio or thermal conductivity ratio.

- Inspecting the worth of using high porosity metal foams in enhancing the heat dissipated by rotating cooling passages proves that this proposal is practically justified and efficient. 


\title{
Developing Convective Flow in a Square Channel Partially Filled with a High Porosity Metal Foam and Rotating in a Parallel-Mode
}

\author{
Ahmed Alhusseny ${ }^{1,2, *}$ and Ali Turan ${ }^{1}$, Adel Nasser ${ }^{1}$ \\ ${ }^{1}$ School of Mechanical, Aerospace and Civil Engineering, The University of Manchester, Manchester, UK \\ ${ }^{2}$ Mechanical Engineering Department, College of Engineering, University of Kufa, Najaf, Iraq
}

\begin{abstract}
The development of three-dimensional heat transfer and fluid flow in a square channel rotating in a parallel-mode has been investigated numerically. The duct is partially occupied by a foam material of high porosity $\varepsilon \geq 0.89$ and subjected to a uniform wall heat flux. In regards to the influence of rotation, both the centrifugal buoyancy and Coriolis forces are considered in the current study. The generalised model is used to mathematically simulate the momentum equations employing the Boussinesq approximation for the density variation. Moreover, thermal dispersion has been taken into account with considering that fluid and solid phases are in a local thermal non-equilibrium. The governing equations are discretised according to the finite volume method with employing a hybrid differencing scheme. Computations are performed for a wide range of parameters including the hollow ratio $(0 \leq \mathrm{S} \leq 1)$, foam porosity $(0.89 \leq \varepsilon \leq 0.97)$, pore density $(5 \mathrm{PPI} \leq \omega \leq 40 \mathrm{PPI})$, solid to fluid thermal conductivity ratio $(250 \leq \kappa \leq 4000)$, Reynolds number $(250 \leq \mathrm{Re} \leq 2000)$, and rotation number $(0 \leq \mathrm{Ro} \leq 1)$, while the values of characteristic temperature difference and Prandtl numbers are maintained constant at $\Delta \mathrm{T}_{\mathrm{c}}=1000^{\circ} \mathrm{C}$ and $\operatorname{Pr}=0.7$, respectively. Results reveal that flow resistance and heat transport are augmented with either decreasing the hollow ratio and foam porosity or increasing Reynolds and rotation numbers, while two contradictory trends are found for the impact of increasing pore density on heat transfer; either enhancing or suppressing depending on the size of hollow zone. In addition, both rotation and thermal dispersion have dominant roles in enhancing heat transfer at the higher levels of porosity or the lower values of conductivity ratios. However, these roles are reduced gradually with decreasing the foam porosity or increasing thermal conductivity ratio, but do not completely vanish. Eventually, the worth of using high porosity fibrous media in enhancing the heat transported through rotating channels has been inspected. An overall enhancement parameter is compared for the current study with a previous work regarding turbulent flow in a rotating clear channel, where it has been confirmed that the current proposal is practically justified and efficient.
\end{abstract}

\section{KEYWORDS: High Porosity Foam, Rotation, Channel, Thermal Dispersion, Partial Occupation, Interface}

\author{
Nomenclature \\ a \\ $a_{s f}$ \\ $c_{p}$ \\ $\boldsymbol{d}_{f}$ \\ $d_{p}$ \\ $\mathrm{Da}$ \\ $D_{h}$ \\ $\boldsymbol{E}$ \\ $\boldsymbol{F}$ \\ $\boldsymbol{h}_{\text {sf }}$ \\ $\boldsymbol{H}$ \\ $\boldsymbol{H}_{\text {sf }}$ \\ $\boldsymbol{k}$ \\ $\boldsymbol{K} \quad$ Permeability of the porous medium \\ *E-mail address: Ahmed.Alhusseny@ postgrad.manchester.ac.uk \\ Side length of the channel \\ Solid-to-fluid interfacial specific surface area \\ Specific heat of fluid phase \\ Fiber diameter \\ Pore diameter \\ Darcy number, $\boldsymbol{D a}=\boldsymbol{K} / \boldsymbol{D}_{\boldsymbol{h}}{ }^{2}$ \\ Hydraulic diameter of the channel \\ Dimensionless eccentricity of the rotating channel $\boldsymbol{E}=\boldsymbol{H} / \boldsymbol{D}_{\boldsymbol{h}}$ \\ Inertial coefficient \\ Solid-to-fluid interfacial specific heat transfer coefficient \\ Radial distance from the axis of rotation to the lower wall of the duct \\ Dimensionless solid-to-fluid interfacial specific heat transfer coefficient \\ Thermal conductivity
}

ined.Alhusseny@ 


\begin{tabular}{|c|c|}
\hline $\mathrm{Nu}$ & Nusselt number \\
\hline$p$ & Dimensional pressure \\
\hline$p_{r}$ & Dimensional reduced pressure \\
\hline $\boldsymbol{P}$ & Dimensionless reduced pressure \\
\hline $\operatorname{Pr}$ & Prandtl Number, $\operatorname{Pr}=v_{f} / \alpha_{e}$ \\
\hline$R a_{\Omega}$ & Rotational Rayleigh number, $R a_{\Omega}=\Omega^{2} H \beta \Delta T_{c} a^{3} / v_{f} \alpha$ \\
\hline $\boldsymbol{R e}$ & Reynolds number, $\boldsymbol{R} \boldsymbol{e}=\boldsymbol{u}_{\text {in }} \boldsymbol{a} / \boldsymbol{v}_{f}$ \\
\hline $\boldsymbol{R} \boldsymbol{e}_{d}$ & Reynolds number based on the fluid velocity near the fiber, $\boldsymbol{R} \boldsymbol{e}_{d}=\boldsymbol{u} \boldsymbol{d}_{f} / \boldsymbol{\varepsilon} \boldsymbol{v}_{f}$ \\
\hline $\operatorname{Re}_{\Omega}$ & Rotational Reynolds number, $\operatorname{Re}_{\Omega}=\Omega D_{h}{ }^{2} / v$ \\
\hline Ro & Rotation number, $R o=\Omega D_{h} / u_{i n}$ \\
\hline$s$ & Hollow region size \\
\hline$S$ & Hollow ratio, $S=s / D_{h}$ \\
\hline$T$ & Dimensional temperature \\
\hline$\Delta T_{c}$ & Dimensional characteristic temperature difference, $\Delta \boldsymbol{T}_{c}=\boldsymbol{q}_{w} \boldsymbol{a} / \boldsymbol{k}_{f e}$ \\
\hline$u, v, w$ & Dimensional velocity components \\
\hline$U, V, W$ & Dimensionless velocity components \\
\hline $\mathbf{v}$ & Dimensional velocity vector \\
\hline $\mathbf{x}$ & Dimensional position vector \\
\hline$x, y, z$ & Dimensional coordinates \\
\hline$X, Y, Z$ & Dimensionless coordinates \\
\hline \multicolumn{2}{|c|}{ Greek symbols } \\
\hline $\boldsymbol{\theta}$ & Dimensionless temperature \\
\hline$\rho_{f}$ & Fluid density \\
\hline $\boldsymbol{\mu}_{f}$ & Dynamic viscosity \\
\hline$v_{f}$ & Kinematic viscosity \\
\hline$\varepsilon$ & Porosity of the fibrous medium \\
\hline $\boldsymbol{\kappa}$ & Solid to fluid-phase thermal conductivity ratio \\
\hline $\boldsymbol{\kappa}_{d}$ & Dispersive to fluid-phase effective thermal conductivity ratio \\
\hline $\mathbf{\Omega}$ & Angular velocity \\
\hline $\boldsymbol{\beta}$ & Coefficient of thermal expansion \\
\hline$\omega$ & Pore density \\
\hline$\xi$ & Mass flow fraction, $\boldsymbol{\xi}=\dot{\boldsymbol{m}}_{\mathbf{p}} /\left(\dot{\boldsymbol{m}}_{\mathbf{p}}+\dot{\boldsymbol{m}}_{\boldsymbol{H}}\right)$ \\
\hline$\gamma$ & Distinguishing parameter between the hollow and porous region \\
\hline \multicolumn{2}{|c|}{ Subscripts } \\
\hline $\boldsymbol{b}$ & Bulk \\
\hline$d$ & Dispersive \\
\hline $\boldsymbol{e}$ & Effective \\
\hline$f$ & Fluid phase \\
\hline $\boldsymbol{H}$ & Hollow region \\
\hline in & Inlet \\
\hline int & Interface surface between the hollow and porous region \\
\hline $\boldsymbol{P}$ & Porous region \\
\hline$s$ & Solid phase \\
\hline$w$, avg & Peripherally wall averaged \\
\hline $\mathbf{\Omega}$ & Rotation \\
\hline Superscr & \\
\hline$n$ & Normal to the interface between the hollow and porous region \\
\hline
\end{tabular}

\section{Introduction}

Rotating machinery operating at extreme temperature conditions usually need to be cooled internally by involving cooling channels inside them. Flow via passages parallel to the axis of rotation, or what is usually called "rotating in a parallel mode", are involved in some of the cooling aspects such as the rotor 
windings of high-capacity electrical generators, which allows for increased magnetic and electrical loadings. The phenomena of fluid flow and heat transfer in stationary channels are considerably different from those in the rotating case due to the existence of Coriolis and centrifugal forces. This is why it is unlikely to apply their empirical correlations and theoretical solutions to the rotating ones Yang et al. [1]. According to its orientation, rotation of channels can be classified into axial, parallel, radial or slant mode Soong [2].

Regarding to convective fluid flow in channels rotating in parallel-mode, Morris [3] presented an extensive review including reported results of analytical and experimental studies for fully developed and developing, laminar and turbulent fluid flow and heat transfer in circular or rectangular channels. Centrifugal buoyancy effect on developing convective laminar flow via a rectangular channel rotating in parallel mode was studied numerically by Neti et al. [4] and then experimentally by Levy et al. [5], where it was found that both pressure drop and heat transfer rate are enhanced noticeably with increasing the rotation rate. The development of secondary flow due to centrifugal buoyancy in channels rotating about a parallel axis was examined numerically by Soong and Yan [6] for both iso-flux and isothermal conditions. It was noticed that rotational effects in the case of constant heat flux are more important than those in the isothermal case, where secondary flow at the fully developed region retains its vortices in the iso-flux channels unlike the isothermal ducts where they almost vanish. Recently, a numerical study of developing turbulent flow and heat transfer in a square channel rotating in parallel mode was conducted by Sleiti and Kapat [7]. The problem was examined for high levels of both rotation and applied heat flux. This study reveals that total heat transfer rate is, in general, enhanced with increasing the rotation rate although it is reduced at the wall closest to the axis of rotation.

Convective flows in porous materials have been investigated widely for over the last decades and various aspects were considered for different applications, where their state of art has been summarised extensively by Nield and Bejan [8]. However, most studies have been limited to granular materials and packed beds, which have a porosity range of $0.3-0.6$. Therefore, there are relatively few studies on convective flow phenomena in materials that have very high porosity $(\varepsilon \geq 0.89)$ like metal foams.

High porosity metal foams are usually porous media with low density and novel structural and thermal properties Tianjian [9]. They offer light weight, high rigidity and strength, and high surface area, which make them able to recycle energy efficiently. Therefore and due to their ability to meet the high rates of thermal dissipation required in electronic industry, they have received more attention recently. Also, their open-cell structure makes them less resistant to the fluids flowing through them, and hence, pressure drop across them is much less than it in the case of flow via packed beds or granular porous media. Forced convection in high porosity metal foams was studied analytically and experimentally by Hunt and Tien [10] with taking into account the effect of thermal dispersion on the heat transport performance. It was found that dispersive transport becomes more significant with increasing the flow rate or the medium permeability. Metal foams are often classified as high porosity materials that consist of irregular shaped and tortuous flow passages. However, some aspects regarding to granular porous media and packed beds need to be adjusted for metal foams Boomsma et al. [11]. Pressure drop and heat transfer through the fibrous medium are significantly affected by its geometrical characteristics such as fiber size, pore size, pore density, and cell shape. A model for the fiber to pore diameter ratio $\left(\mathrm{d}_{\mathrm{f}} / \mathrm{d}_{\mathrm{p}}\right)$ was developed by Calmidi [12] as a function of the foam porosity. Furthermore, this experimental and analytical study proposed mathematical models for both permeability and inertial coefficient as functions of the fiber and pore diameters in addition to the porosity. Due to the significant difference existed between thermal conductivities of metal foams and fluid flowing across them, using the twoequations model for tracking heat transport is indispensable. Many fundamental works were conducted for investigating the influence of both the interfacial surface area and heat transfer coefficient on the heat exchange between the fluid and solid phases in addition to developing a more realistic implementation for thermal boundary conditions at the walls, where valuable and detailed discussions can be found in the analytical works presented by [13-17]. A theoretical model for the effective thermal 
conductivity of high porosity metal foams was derived by Calmidi and Mahajan [18], where it was matching well with the experimental data obtained for both air and water as a fluid-phase. Later, the same authors conducted a numerical study, [19], for forced convection in a rectangular channel filled with high porosity fiber, where they used the effective thermal conductivity model derived earlier to analyse the impact of thermal dispersion and local thermal non-equilibrium on heat transport performance. It was found that their model is quite sufficient for simulating convective fluid flow in high porosity metal foams. Studies presented in the field of heat and fluid flow within high porosity fibers were reviewed extensively by Mahjoob and Vafai [20] and then by Zhao [21], where various models regarding convective flow in metal foams were summarised.

In regards to combined fluid flow and heat transfer in rotating porous media, relevant studies have been motivated by its wide range of practical and fundamental applications in engineering and geophysics. Chemical processing, materials, and food processing industries, in addition to rotating machinery are just a few examples of its engineering applications cited by Vadasz [22]. A threedimensional isothermal fluid flow in a rotating square channel occupied by a heterogeneous porous medium was studied analytically by Vadasz [23] and numerically by Havstad and Vadasz [24] using Darcy formulation. The data has confirmed the ability to induce a mainstream flow along the channel by means of the secondary circulation resulting from a locally varying permeability. Natural convection induced by centrifugal acceleration in a narrow porous layer subjected to rotation was examined analytically by Vadasz [25-27] for an axis of rotation attached to, distant from, and located within the porous layer, respectively. The results indicated that displacing the porous layer away from the axis of rotation has a destabilizing effect that enhances the centrifugal buoyancy. Recently, Alhusseny and Turan [28] presented a numerical study for Coriolis' effect on combined heat and mass transfer in a radially rotating porous channel. It was found that rotation has a negative impact on heat and mass transport, where this role is gradually reduced with decreasing the medium permeability, but does not completely vanish. Utilizing metal foams to improve the internal cooling of turbine blades was examined experimentally by Tzeng et al. [29] through filling a radially rotating serpentine channel with aluminum foam. It was found that this proposal enhances the amount of heat transported between the solid and fluid phases, and hence, improves the overall efficiency of cooling process. More recently, Alhusseny and Turan [30] and Alhusseny et al. [31] studied numerically the thermally and hydrodynamically developing flow through a channel rotating in a parallel-mode and fully filled with high porosity metal foam, where effects of rotation, geometrical and thermal characteristics of the fiber, channel aspect ratio, and thermal dispersion on heat transfer performance were investigated.

Despite the fact that using porous media, which have high thermal conductivity, improves heat transport considerably, it also results in relatively high pressure losses. Therefore, it is essential to avoid unjustified pressure drop with keeping the heat transfer at as high rates as possible. To this end, channels partially filled with permeable materials have been proposed, where plenty of investigations have been presented. However, assumption of a local thermal equilibrium existence between fluid and solid phases was considered in the most of former analytical and numerical works conducted. To name a few, the analytical studies presented by $[32,33]$ as well as the numerical works done by $[34,35]$. Due to the significant difference existed between the thermal conductivities of fluid and solid phases in some porous media, e.g. metal foams, the two-equations model becomes indispensable, where a local thermal non-equilibrium occurs. By using the interface boundary conditions proposed by Ochoa-Tapia and Whitaker [36], an analytical study for fully developed convective flow in a channel partially filled with metal foam was conducted by $\mathrm{Xu}$ et al. [37], where local thermal non-equilibrium was taken into account.

Overall, the influence of partially filling the rotating channels with high porosity metal foams has not been examined before. Therefore, the current work aims to present a comprehensive study for the aforementioned proposal and investigating its impact on heat and fluid flow performance. 


\section{Mathematical formulation}

A square channel partially filled with a saturated porous medium and rotating about an axis parallel to the main flow direction "a parallel-mode rotation", as shown in Fig.(1), is assumed to carry a steady, incompressible, and laminar fluid flow within it. Both the channel length and radial distance from the lower wall of the channel to the axis of rotation have been equal to 32 times the duct hydraulic diameter. All the lateral walls are considered impermeable and subjected to a uniform wall heat flux.

Fig.(1)

The porous material is considered isotropic, homogeneous, and rigid fibrous medium of high porosity $\varepsilon \geq 0.89$. The generalised model is employed to simulate the momentum equations considering both the solid and fluid phases to be in local thermal non-equilibrium with each other. The gravitational effect has been ignored as compared to the rotational buoyancy as suggested by Soong and Yan [6]. The working fluid is air; so, it is possible to neglect the thermal dispersion due to its low effect compared to the relatively high thermal conductivity of the solid matrix as proposed by Calmidi and Mahajan [19]. However, thermal dispersion has been reconsidered in the current analysis to investigate its impact on the heat and flow fields. Both fluid and solid properties are assumed to be constant everywhere except the fluid density in the centrifugal buoyancy term, which varies according to the Boussinesq approximation. Thus, the dimensional forms of conservation equations of mass, momentum, and energy for both fluid and solid-phase are:

$$
\begin{aligned}
& \nabla \cdot \mathbf{v}=0 \\
& \frac{\rho_{f}}{\varepsilon^{2}}(\mathbf{v} \cdot \nabla) \mathbf{v}=-\nabla p_{r}+\frac{\mu_{f}}{\varepsilon} \nabla^{2} \mathbf{v}-\gamma\left(\frac{\mu_{f}}{K} \mathbf{v}+\rho_{f} \frac{F}{\sqrt{K}}|\mathbf{v}| \mathbf{v}\right)+\left(\rho_{f}-\rho_{0}\right) \Omega^{2}\left(e_{\Omega} \times \mathbf{x}\right) \cdot\left(e_{\Omega} \times \mathbf{x}\right) / 2-\frac{2 \rho_{f} \Omega}{\varepsilon} e_{\Omega} \times \mathbf{v} \\
& \rho_{f} c p_{f}(\mathbf{v} \cdot \nabla) T_{f}=(1-\gamma) k_{f} \nabla^{2} T_{f}+\gamma\left[\left(k_{f e}+k_{d}\right) \nabla^{2} T_{f}+a_{s f} h_{s f}\left(T_{s}-T_{f}\right)\right] \\
& 0=k_{s e} \nabla^{2} T_{s}+a_{s f} h_{s f}\left(T_{f}-T_{s}\right)
\end{aligned}
$$

In Eqs.[(2)-(3)], $\gamma$ is a distinguishing parameter equals to either zero or one depending on whether the flow takes place in the hollow or porous region, respectively; while the medium porosity $\varepsilon$ equals to one just in the hollow region. Also, $\mathrm{p}_{\mathrm{r}}=\mathrm{p}-\rho_{0} \Omega^{2}\left(\mathrm{e}_{\Omega} \times \mathbf{x}\right) \cdot\left(\mathrm{e}_{\Omega} \times \mathbf{x}\right) / 2$ is the reduced pressure generalised to include the constant components of the centrifugal terms, while $\mathrm{K}$ and $\mathrm{F}$ represent the permeability and inertia coefficient of the metal foam, respectively, where their values are computed according to the model proposed by Calmidi [12]:

$$
\begin{aligned}
& K=0.00073 d_{p}^{2}(1-\varepsilon)^{-0.224}\left(\frac{d_{f}}{d_{p}}\right)^{-1.11} \\
& F=0.00212(1-\varepsilon)^{-0.132}\left(\frac{d_{f}}{d_{p}}\right)^{-1.63}
\end{aligned}
$$

The fiber and pore diameters can be estimated as functions of pore density $\omega$ and foam porosity $\varepsilon$, respectively, Calmidi [12]: 


$$
\begin{aligned}
& d_{p}=0.0254 / \omega \\
& d_{f}=1.18 d_{p} \sqrt{\frac{(1-\varepsilon)}{3 \pi}} \frac{1}{1-e^{-(1-\varepsilon) / 0.04}}
\end{aligned}
$$

In addition, $\mathrm{k}_{\mathrm{fe}}$ and $\mathrm{k}_{\mathrm{se}}$ are the effective thermal conductivity of fluid and solid phase, respectively. Their values are computed using the model derived by Calmidi and Mahajan [18]. The dispersion conductivity $\mathrm{k}_{\mathrm{d}}$, which is assumed to be isotropic, is determined based on the work presented by Hunt and Tien [10]:

$k_{d}=C_{d} \rho c p u \sqrt{K}$

Where $C_{d}$ is the coefficient of thermal dispersion and its value was found to be 0.06 as proposed by Calmidi and Mahajan [19]. Finally, $h_{s f}$ and $a_{s f}$ are the solid-fluid interfacial heat transfer coefficient and specific surface area and can be determined using the model suggested by Calmidi and Mahajan [19]:

$$
\begin{aligned}
& N u_{s f}=\frac{h_{s f} d_{f}}{k_{f}}=0.52 \operatorname{Re}_{d}^{0.5} \operatorname{Pr}^{0.37} \\
& a_{s f}=\frac{3 \pi d_{f}}{\left(0.59 d_{p}\right)^{2}}\left[1-e^{-(1-\varepsilon) / 0.04}\right]
\end{aligned}
$$

Although the governing equations of fluid flow and heat transport either within the metal foam or in the hollow zone are not coupled with each other, they are linked together through the interface surface between the two regions. So, Eqs.(1-4) need to be closed by means of interfacial coupling conditions, where continuity of velocity, shear stress, fluid temperature, and heat flux along the fluid-solid interface must be ensured to get meaningful physics, $\mathrm{Xu}$ et al. [37]. To this end, boundary conditions proposed by Ochoa-Tapia and Whitaker [36] for the fluid-solid interface are used with taking the thermal dispersion into account as:

$$
\begin{aligned}
& \left.\mathbf{v}\right|_{\text {int }_{\mathrm{H}}} ^{\mathrm{n}}=\left.\mathbf{v}\right|_{\text {int }_{\mathrm{P}}} ^{\mathrm{n}} \quad(11 . \mathrm{a}) \\
& \left.\mu_{f} \nabla \mathbf{v}\right|_{\text {int }_{H}} ^{n}=\left.\frac{\mu_{f}}{\varepsilon} \nabla \mathbf{v}\right|_{\text {nnt }_{P}} ^{n} \quad(11 . b) \\
& \left.\mathrm{T}_{\mathrm{f}}\right|_{\text {int }_{\mathrm{H}}} ^{\mathrm{n}}=\left.\mathrm{T}_{\mathrm{f}}\right|_{\text {int }_{\mathrm{P}}} ^{\mathrm{n}} \\
& \left.k_{f} \nabla T_{f}\right|_{\text {int }_{H}} ^{n}=\left.\left(\left(k_{f e}+k_{d}\right) \nabla T_{f}+k_{s e} \nabla T_{s}\right)\right|_{\text {int }_{P}} ^{n} \\
& \left.k_{s e} \nabla T_{s}\right|_{\text {int }_{P}} ^{n}=h_{s f}\left(\left.T_{s}\right|_{\text {int }_{P}} ^{n}-\left.T_{f}\right|_{\text {int }_{H}} ^{n}\right)
\end{aligned}
$$

Because the value of Peclet number in the current study is large enough (Pe>100), the downstream effects on momentum and energy balance are small and there is no back-flow expected; hence, the axial diffusion were neglected as recommended by Soong and Yan [6]. Therefore, above governing equations can be rewritten in their detailed dimensionless forms as:

\section{Continuity equation:}

$$
\frac{\partial U}{\partial X}+\frac{\partial V}{\partial Y}+\frac{\partial W}{\partial Z}=0
$$

\section{$X$-Momentum equation:}

$\frac{1}{\varepsilon^{2}}\left(U \frac{\partial U}{\partial X}+V \frac{\partial U}{\partial Y}+W \frac{\partial U}{\partial Z}\right)=-\frac{\partial \bar{P}}{\partial X}+\frac{1}{\varepsilon \operatorname{Re}}\left(\frac{\partial^{2} U}{\partial Y^{2}}+\frac{\partial^{2} U}{\partial Z^{2}}\right)-\gamma\left(\frac{U}{D a \operatorname{Re}}+\frac{F|\mathbf{V}| U}{\sqrt{D a}}\right)$

\section{Y-Momentum equation:}

$\frac{1}{\varepsilon^{2}}\left(U \frac{\partial V}{\partial X}+V \frac{\partial V}{\partial Y}+W \frac{\partial V}{\partial Z}\right)=-\frac{\partial P}{\partial Y}+\frac{1}{\varepsilon \operatorname{Re}}\left(\frac{\partial^{2} V}{\partial Y^{2}}+\frac{\partial^{2} V}{\partial Z^{2}}\right)-\gamma\left(\frac{V}{D a \operatorname{Re}}+\frac{F|\mathbf{V}| V}{\sqrt{D a}}\right)-\frac{R a_{\Omega}}{\operatorname{Pr}^{2} \operatorname{Re}^{2}}\left(\frac{Y}{E}+1\right) \theta_{f}+\frac{2 R o W}{\varepsilon}$ 


\section{Z-Momentum equation:}

$\frac{1}{\varepsilon^{2}}\left(U \frac{\partial W}{\partial X}+V \frac{\partial W}{\partial Y}+W \frac{\partial W}{\partial Z}\right)=-\frac{\partial P}{\partial Z}+\frac{1}{\varepsilon \operatorname{Re}}\left(\frac{\partial^{2} W}{\partial Y^{2}}+\frac{\partial^{2} W}{\partial Z^{2}}\right)-\gamma\left(\frac{W}{D a \operatorname{Re}}+\frac{F|\mathbf{V}| W}{\sqrt{D a}}\right)-\frac{R a_{\Omega}}{\operatorname{Pr}^{2}}\left(\frac{Z}{E}-\frac{1}{2 E}\right) \theta_{f}-\frac{2 \operatorname{RoV}}{\varepsilon}$

Fluid-Phase Energy Equation:

$U \frac{\partial \theta_{f}}{\partial X}+V \frac{\partial \theta_{f}}{\partial Y}+W \frac{\partial \theta_{f}}{\partial Z}=\frac{1}{\operatorname{Pr} \operatorname{Re}}\left[\left((1-\gamma) \frac{k_{f}}{k_{f e}}+\gamma\left(1+\kappa_{d}\right)\right)\left(\frac{\partial^{2} \theta_{f}}{\partial Y^{2}}+\frac{\partial^{2} \theta_{f}}{\partial Z^{2}}\right)+\gamma H_{s f}\left(\theta_{s}-\theta_{f}\right)\right]$

Solid-Phase Energy Equation:

$0=\frac{\partial^{2} \theta_{f}}{\partial Y^{2}}+\frac{\partial^{2} \theta_{f}}{\partial Z^{2}}+\frac{H_{s f}}{\kappa_{e}}\left(\theta_{f}-\theta_{s}\right)$

The non-dimensional parameters employed in Eqs.(12-17) are:

$X=\frac{x}{D_{h}}, \quad Y=\frac{y}{D_{h}}, \quad Z=\frac{z}{D_{h}}$

$U=\frac{u}{u_{i n}}, \quad V=\frac{v}{u_{i n}}, \quad W=\frac{w}{u_{i n}}$,

$P=\frac{p_{r}}{\rho_{f} u_{i n}^{2}}, \quad \theta_{f}=\frac{T_{f}-T_{i n}}{\Delta T_{c}}, \quad \theta_{s}=\frac{T_{s}-T_{i n}}{\Delta T_{c}}$,

$\operatorname{Pr}=\frac{v_{f}}{\alpha_{e}}, \quad \operatorname{Re}=\frac{u_{i n} D_{h}}{v_{f}}, \quad \operatorname{Ro}=\frac{\Omega D_{h}}{u_{i n}}, \quad R a_{\Omega}=\frac{\Omega^{2} H \beta \Delta T_{c} D_{h}^{3}}{v_{f} \alpha_{e}}$

$D a=\frac{K}{D_{h}^{2}}, \quad \kappa_{e}=\frac{k_{s e}}{k_{f e}}, \quad \kappa_{d}=\frac{k_{d}}{k_{f e}}, \quad H_{s f}=\frac{a_{s f} h_{s f} a^{2}}{k_{f e}}$

Note that $\mathrm{H}_{\mathrm{sf}}$ is note a constant as it depends on the value of $\mathrm{h}_{\mathrm{sf}}$, which varies with the local value of the velocity according to Eq.(9), while $\alpha_{\mathrm{e}}$ refers to the thermal diffusivity of the fluid-phase depending on its effective thermal conductivity $\mathrm{k}_{\mathrm{fe}}$. As the flow is assumed to be thermally and hydrodynamically developing, the velocity and temperature profiles are considered uniform at the channel inlet. Moreover, the no-slip condition is applied at the walls in addition to assuming that the heat flux prescribed at channel walls is divided between the fluid and solid phases depending on their effective thermal conductivities and temperature gradients at the channel walls, Alazmi and Vafai [15]. Hence, the dimensionless forms of boundary conditions employed to solve the aforementioned equations are:

$$
\left.\begin{array}{l}
U=1 \& V=W=0, \quad \theta_{f}=\theta_{s}=0 \quad \text { at } X=0 \\
U=V=W=0, \frac{\partial \theta_{f}}{\partial Y}+\kappa_{e} \frac{\partial \theta_{s}}{\partial Y}=-1,1 \quad \text { at } Y=0,1 \\
U=V=W=0, \frac{\partial \theta_{f}}{\partial Z}+\kappa_{e} \frac{\partial \theta_{s}}{\partial Z}=-1,1 \quad \text { at } Z=0,1
\end{array}\right\}
$$

Eventually, the heat transfer rate is measured by means of local Nusselt number along the axial direction, where its values are computed according to the peripheral average of the fluid-phase temperatures at the wall in addition to the fluid bulk temperature as follow:

$$
N u_{l}=\frac{1}{\theta_{w, a v g}-\theta_{f, b}}
$$




\section{Solution procedure}

Eqs.(12-17) were discretised and transformed into linear algebraic forms by using the finite volume method. The pressure gradient at the axial direction is decoupled from its gradients at the cross-sectional directions by assuming that the flow is parabolic and employing the solution procedure of Patankar and Spalding [38]. A hybrid differencing approximation developed by Spalding [39] was employed to represent the convection terms in the transverse directions. By implementing this scheme, either secondorder accurate solution at low Peclet number or stable solution at corresponding high values is ensured. Solution of the resulting discretised algebraic equations was done via the tri-diagonal matrix algorithm. To predict potentially steep gradients of velocity and temperatures at the channel inlet, porous-fluid interface, and channel walls more precisely, the mesh was refined in the axial direction at the entrance region according to the method proposed by Hoffmann and Chiang [40]; while more grid points have been clustered in the vicinity of the porous-fluid interface as well as the walls boundaries symmetrically by implementing the practices of Havstad and Vadasz [24]. The dependence of numerical results on the grid size was checked by examining six sets of mesh for each hollow ratio mentioned in Table(1) at $\operatorname{Re}=2000, \operatorname{Ro}=0.5, \operatorname{Pr}=0.7, \varepsilon=0.89, \omega=5 \mathrm{PPI}$, and $\kappa=1000$. It has been noticed that the deviation in the mean Nusselt number value becomes less than $1 \%$ between the fourth and the fifth set of grid and thereafter. Accordingly, the fourth set of mesh can be considered sufficient for accuracy of the current study. However, it is noticed that the mesh required for the partially filled channel, $S>0$, has a higher size than it for the fully occupied one, $S=0$, due to the immediate and rapid change in the velocity and temperature fields that occurs at the porous-fluid interface regions. Convergence was measured in terms of the maximum change allowed in each variable during any iteration, where its maximum value for convergence check was $10^{-6}$. An in-house FORTRAN code was built to solve the current problem and validated with some of the previous experiments related to the present study. Fig.(2.a) shows a comparison between the experimental data of excess temperature of air over ambient obtained by Kamath et al. [41] for mixed convection through a vertical channel filled with a high porosity foam and those computed by the current code. In regards to the rotational effects, the variation of mean Nusselt number with rotation level was validated against the experiments conducted by Morris [3] for the developing fluid flow and heat transfer in a clear square channel subjected to parallel-mode rotation as shown in Fig.(2.b). It is obvious that the computed results by the current code are in a good agreement with the earlier studies, although there were small variances due to the approximations in the present numerical analysis and the probable inaccuracy in the experimentally measured data.

Table(1) The variation of Nusselt number with the grid size at different hollow ratios at $\mathrm{Ro}=0.5$

\begin{tabular}{|c|c|c|c|c|c|c|}
\hline \multirow{2}{*}{$S=0$} & $450 \times 30 \times 30$ & $500 \times 40 \times 40$ & $550 \times 50 \times 50$ & $600 \times 60 \times 60$ & $650 \times 70 \times 70$ & $700 \times 80 \times 80$ \\
\cline { 2 - 7 } & 80.723 & 91.774 & 99.465 & 102.777 & 103.226 & 103.628 \\
\hline \multirow{2}{*}{$S=0.2$} & $450 \times 50 \times 50$ & $500 \times 60 \times 60$ & $550 \times 70 \times 70$ & $600 \times 80 \times 80$ & $650 \times 90 \times 90$ & $700 \times 100 \times 100$ \\
\cline { 2 - 7 } & 79.497 & 89.342 & 96.727 & 100.224 & 100.731 & 101.105 \\
\hline \multirow{2}{*}{$S=0.4$} & $450 \times 50 \times 50$ & $500 \times 60 \times 60$ & $550 \times 70 \times 70$ & $600 \times 80 \times 80$ & $650 \times 90 \times 90$ & $700 \times 100 \times 100$ \\
\cline { 2 - 7 } & 69.346 & 80.165 & 87.572 & 91.761 & 92.384 & 92.643 \\
\hline \multirow{2}{*}{$S=0.6$} & $450 \times 50 \times 50$ & $500 \times 60 \times 60$ & $550 \times 70 \times 70$ & $600 \times 80 \times 80$ & $650 \times 90 \times 90$ & $700 \times 100 \times 100$ \\
\cline { 2 - 7 } & 55.254 & 65.561 & 73.119 & 76.103 & 76.675 & 76.971 \\
\hline \multirow{2}{*}{$S=0.8$} & $450 \times 50 \times 50$ & $500 \times 60 \times 60$ & $550 \times 70 \times 70$ & $600 \times 80 \times 80$ & $650 \times 90 \times 90$ & $700 \times 100 \times 100$ \\
\cline { 2 - 7 } & 37.376 & 46.214 & 52.675 & 55.276 & 55.702 & 55.936 \\
\hline
\end{tabular}

Fig.(2) 


\section{Results}

The obtained results are presented for a wide range of parameters used, which are the hollow ratio $(0 \leq \mathrm{S} \leq 1)$, foam porosity $(0.89 \leq \varepsilon \leq 0.97)$, pore density $(5 \mathrm{PPI} \leq \omega \leq 40 \mathrm{PPI})$, solid to fluid thermal conductivity ratio (250 $\leq \kappa \leq 4000)$, Reynolds number $(250 \leq \mathrm{Re} \leq 2000)$, and rotation number $(0 \leq \mathrm{Ro} \leq 1)$, while the values of characteristic temperature difference and Prandtl number are kept constant at $\Delta \mathrm{T}_{\mathrm{c}}=1000^{\circ} \mathrm{C}$ and $\operatorname{Pr}=0.7$, respectively.

\section{1- Combined influence of rotation and hollow ratio on heat and fluid flow development}

Effects of the hollow region size accompanied with rotation on the development of flow field are illustrated in Figs.(3.a-e) for various axial locations at $\varepsilon=0.97, \omega=10 \mathrm{PPI}, \kappa=1000, \mathrm{Re}=2000$, and $\mathrm{Ro}=0.5$. At the channel inlet $X=0$, the flow patterns develop as the standard hydrodynamic boundary layer, while at $\mathrm{X}=1$ and thereafter, influence of body forces induced by rotation starts to appear, resulting in forming transverse vortices and reorganising the flow patterns in a quite different way from the classical flow behavior. More downstream, the transverse velocity vectors show a typical behavior of mixed convection, where the fluid is rising along the hot walls and against the centrifugal body force creating two main vortices along the leading and trailing walls, which then are shifted upward with further development of the flow field. Thus, boundary layer at the upper wall becomes thinner than it at the remaining walls in contrast to it at the lower wall, which is clearly thicker. Also, it is obvious that increasing the hollow region size makes the boundary layer much thinner at the upper wall and much thicker at the lower wall. The reason of this is the reduction occurred in the overall resistance of the porous layer to the fluid flowing through it. As a result, heat accumulates at the lower wall causing it to be hotter unlike the upper wall, which is cooler than the remaining walls as shown in Fig.(4). Moreover, it is clear that increasing the size of hollow region reduces the amount of heat absorbed by the fluid in addition to increasing heat accumulation at the lower wall, while it results in reducing the temperature at the remaining walls to levels even lower than the corresponding bulk temperatures. Overall, enlarging the hollow zone results in reducing the mass flow rate in the foam region; and hence, decreasing the heat transported to the fluid and pressure drop resulted as shown in Fig.(5). At the aforementioned rotation level, the dominance of centrifugal buoyancy effects at the smaller values of hollow ratios, e.g. $\mathrm{S} \leq 0.5$, is obvious from the symmetry of the flow patterns around the mid-vertical axis $\mathrm{Z}=0.5$, which overshadows the influence of Coriolis forces completely as shown in Figs.(3.a-c). Increasing the hollow region size over the aforementioned level causes breaking down the symmetry phenomenon due to the relative reduction in the centrifugal buoyancy compared to Coriolis forces, which become more noticeable and influential as noticed in Figs.(3.d,e).

Fig.(3)

Fig.(4)

Fig.(5) 


\section{2- Combined influence of foam geometrical properties on heat and fluid flow patterns}

Combined effects of the hollow region size, foam porosity, and pore density accompanied with rotation on the development of fluid flow and heat transfer can be explained firstly by examining the mass flow fraction in the porous region $\xi$. Its values at $X=32, \kappa=1000, R e=2000$, and $\operatorname{Ro}=0.5$ are illustrated in Fig.(6) for various levels of the above mentioned parameters. As expected, $\xi$ decreases with increasing the hollow ratio as a result of the reduction occurred in the porous layer size. Meanwhile, increasing pore density $\omega$ leads to decrease both pore and fiber diameters, and hence, the foam permeability is reduced as well, Eq.(5), resulting in augmenting the fiber resistance to the fluid flowing across it. This means that more fluid particles are forced to migrate from the porous to the open zone, which is far less resistant to the fluid flow. In contrast, increasing the porosity has a positive impact on the mass flow fraction as it makes the fiber ligaments thinner at a fixed pore density, which results in reducing the effective volume of the obstructing solid and the flow resistance as well.

Fig.(6)

To further clarify the aforementioned effects on the flow development, longitudinal velocity profiles are plotted at the mid-vertical axis $Z=0.5$ for two axial locations, $X=1$ and $X=32$, and compared for various hollow ratios as shown in Figs.(7). For the partially filled channel $S>0$, the flow is clearly heterogeneous because of the sharp change in the permeability, which occurs suddenly at the porous-fluid interface. This flow heterogeneity is much more obvious at the higher levels of pore density as a result of the additional reduction happened in the foam permeability, which leads to create a much higher discrepancy between the porous and open regions. As mentioned earlier, and due to the centrifugal buoyancy and Coriolis force, flow velocity at $X=32$ is significantly accelerated in the upper part of the channel unlike the lower part, where it is clearly decelerated. However, the role played by rotation is apparently less dominant for the lower values of hollow ratio due to the increase in the volume of the solid matrix, which acts as an obstructing or damper to the flow. Moreover, increasing pore density has a significant impact on promoting the flow velocity in the clear region and suppressing it in the porous region. This role can be clarified more in Figs.(8.a,b), where the development of axial velocity and fluid temperature are compared for various levels of pore density. Due to the rotation impact mentioned earlier, the upper wall becomes, in general, cooler than the lower wall. In the meantime, the solid matrix obstruction to the fluid flow is augmented considerably with increasing pore density, and hence, rotation effects at the porous region are damped as well.

Fig.(7)

Fig.(8) 
Eventually, combined effect of porosity and pore density on velocity and temperature profiles can be illustrated in Fig.(9.a,b) for two porosity levels and various values of pore density. Unlike its impact on velocity profiles, which looks marginal compared to the influence of pore density; porosity affects the fluid temperature profiles significantly, where its decrease results in improving heat transport considerably due to the significant augmentation in the overall thermal conductivity of the foam.

Fig.(9)

Overall, combined effects of hollow ratio, porosity, and pore density on both heat transfer performance and total pressure drop are illustrated in Figs.(10.a,b), respectively. Heat transfer and pressure drop are augmented with reducing the size of hollow region due to increasing the volume occupied by the fluidsaturated matrix. Similarly, reducing porosity enhances heat transport as a result of augmenting the effective thermal conductivity and causes more pressure drop for the same reason mentioned above. In contrast, decreasing pore density leads to a considerable reduction in the pressure loss especially at the lower hollow ratios. Moreover, Nusselt number does not change monotonically with pore density. Instead, there are two incompatible or contradictory trends, where a transmutation point is existed. In general, increasing pore density enhances heat transfer at the lower values of hollow ratio, e.g. $\mathrm{S}<0.1$; while it reduces heat transport for the larger sizes of hollow region, e.g. $\mathrm{S} \geq 0.4$. This can be attributed to the fact that although increasing pore density leads to augmenting the surface area of the solid matrix, which improves heat transfer; it amplifies the solid resistance to the fluid flow, which reduces the mass flow rate across the solid matrix at the higher hollow ratios; and hence, reduces the heat transport rate.

\section{Fig.(10)}

\section{3- Effects of rotation rate on heat and fluid flow}

To explain the effects of rotation level on the flow and heat transfer patterns, the axial velocity contours and transverse velocity vectors are presented at $\mathrm{X}=32, \mathrm{~S}=0.6, \varepsilon=0.97, \omega=10 \mathrm{PPI}, \kappa=1000$, $\mathrm{Re}=2000$ and for various rotation levels as shown in Figs.(11.a-d). It is noticed that increasing the rotation rate enhances the strength of transverse vortices, and hence, makes the boundary layer much thicker at the lower wall and much thinner at the upper wall. As a result, the overall heat transfer rate and total pressure drop is augmented considerably as illustrated in Fig.(12), which displays how increasing the rotation rate improves heat transport, but with costing more pressure loss. In addition, it is obvious that increasing the size of hollow region or foam porosity leads to augmenting the role played by rotation rate and makes it more influential. In general, increasing either rotation strength or hollow ratio or decreasing the foam porosity results in improving the overall system performance of channels fully or partially occupied with metal foams as shown in Fig.(13), which illustrates how rotation rate affects the overall enhancement factor $(\mathrm{Nu} / \Delta \mathrm{P})$ for different values of hollow ratio and medium porosity at $\omega=5 P P I, \kappa=1000$, and $\mathrm{Re}=2000$. 
Fig.(11)

Fig.(12)

Fig.(13)

\section{4- Combined influence of thermal conductivity ratio and dispersion on heat transfer rate}

Intra-pore mixing that occurs when fluid penetrates across solid particles, which is a hydrodynamic and non-Darcian phenomenon, is called thermal dispersion Hunt and Tien [10]. Its effect on heat transfer performance at $\omega=5$ PPI and $\mathrm{Ro}=0.5$ is explained in Figs.(14.a,b) for two values of foam porosity. According to Eq.(3), heat transported to the fluid-phase by conduction is improved when thermal dispersion is not ignored. Therefore, Nusselt number levels are generally higher in the case of taking it into account. It is also clear that heat transfer augmentation by dispersive transport is more significant at the higher levels of porosity or the lower values of hollow ratio and thermal conductivity ratios. The reason for this is that dispersion prevails and becomes more obvious when the overall thermal conductivity of the porous medium is relatively low and the flow velocity is high enough; and hence, the thermal conductivity of the fluid-phase is considerably improved allowing more heat to be gained by the fluid particles. In addition, increasing the medium porosity has two implied contributions in enhancing the role played by thermal dispersion. The first, permeability increases directly with the foam porosity leading to augmenting the dispersive thermal conductivity according to Eq.(8). Secondly, it results in decreasing the effective thermal conductivity of the solid-phase, which makes the influence of thermal dispersion more obvious on transferring heat to the fluid-phase.

\section{Fig.(14)}

\section{5- Overall Enhancement}

Using porous media to enhance heat transfer in practical applications is sometimes criticised in that it relatively causes more cost than its gain, or in other words it consumes more pumping power than the heat transferred within it. Therefore, it needs to be known whether it is worth using metal foams in the current problem or not. To do so, the overall enhancement factor used earlier by the authors Alhusseny and Turan [30] is re-employed in the current analysis, which is defined as the ratio of heat transported to the pumping power $\mathrm{Nu} /(\operatorname{Re} \Delta \mathrm{P}$ ) (or the ratio of gain to the cost). So, as long as this factor is higher, the 
overall efficiency is better. To investigate the impact of Reynolds number on the overall performance obtained, the mean Nusselt number and overall enhancement factor are plotted at various values of Reynolds number and for different hollow ratios as shown in Fig.(15.a). For the channel fully or partially filled with metal foam, it is obvious that increasing Reynolds number enhances the heat transported, but in the meantime it causes higher pressure drop. Overall, the performance is improved significantly with increasing Reynolds number unlike the flow case through a clear channel $S=1$, where it clearly deteriorates. To confirm this outcome, a comparison was done between the data obtained by Sleiti and Kapat [7] for turbulent air flow at $\mathrm{Re}=20,000$ in a clear square channel rotating in parallelmode and the current data of laminar air flow at $\mathrm{Re}=2000$ through a channel occupied with metal foam. The same dimensions, wall heat flux, and rotation rates were used, where $L / D_{h}=64, H / D_{h}=32, q_{w}=6.45$ $\mathrm{KW} / \mathrm{m}^{2}$, and $\mathrm{Re}_{\Omega}=0,1260,2800$, and 7000. The comparison shown in Fig.(15.b) reveals that the overall enhancement by pumping a laminar flow through a channel rotating about a parallel axis and fully or partially occupied with a high porosity fiber is quite better than the performance of turbulent flow in a similar but clear channel, especially at the higher values of rotational speed. Moreover, the overall performance obtained at the higher values of hollow ratio, e.g. $\mathrm{S} \geq 0.4$, is much better than it at the lower values $S \leq 0.2$. However, the best performance depends on the quantity of heat needs to be dissipated by the fluid flow regardless of the pressure loss resulted. Therefore, it is crucial to select an appropriate size of hollow zone to achieve the best performance depending on the design conditions required. Overall, using a high porosity metal foam in the current problem is practically justified as it has the ability to meet the high thermal demands without producing an expensive pressure drop such as is found in packed beds or granular porous media.

\section{Fig.(15)}

\section{Conclusions}

A numerical study for the development of three-dimensional heat and fluid flow in a square channel rotating about a parallel axis has been presented. The duct is partially occupied by a metal foam of high porosity and subjected to a uniform wall heat flux. Both centrifugal buoyancy effect and Coriolis forces are considered regarding to rotation effects in the current study. The generalised model is used to simulate the momentum equations employing the Boussinesq approximation for the density variation. Moreover, thermal dispersion is taken into account with considering that fluid and solid phases are in local thermal non-equilibrium. The data obtained are presented in terms of a wide range of parameters used including the hollow ratio, foam porosity, pore density, solid to fluid thermal conductivity ratio, rotation number, and Reynolds number. Heat transport and pressure drop are clearly augmented with either decreasing the hollow ratio and foam porosity or increasing the rotation and Reynolds numbers. Two contradictory trends are found for the impact of increasing pore density on heat transfer; either enhancing or suppressing depending on whether the hollow ratio is very low $S<0.1$ or high enough $\mathrm{S} \geq 0.4$, respectively, while the pressure drop increases with pore density. The results also reveal that both rotation and thermal dispersion have dominant roles in enhancing heat transfer at the higher levels of porosity or the lower values of thermal conductivity ratios. However, these roles are reduced gradually with decreasing foam porosity or increasing thermal conductivity ratio, but do not completely vanish. Eventually, the worth of using high porosity metal foams in enhancing the heat transported through rotating channels has been inspected. An overall enhancement parameter is compared for the current study with a previous work regarding turbulent flow in a rotating clear channel, where it has been confirmed that the current proposal is practically justified and efficient. 


\section{Acknowledgements}

The financial support from the Higher Committee for Education Development in Iraq and the University of Kufa is greatly acknowledged.

\section{References}

[1] W.-J. Yang, , S. Fann, J.H. Kim, Heat and fluid flow inside rotating channels, ASME Appl. Mech. Rev. 47 (8) (1994) 367-396.

[2] C.Y. Soong, Thermal buoyancy effects in rotating non-isothermal flows, Int. J. Rotating Machinery 7

(6) (2001) 435-446.

[3] W.D. Morris, Heat transfer and fluid flow in rotating coolant channels, Research Studies Pr./John Wiley and Sons, Chichester, 1981.

[4] S. Neti, A.S. Warnock, E.K. Levy, K.S. Kannan, Computation of laminar heat transfer in rotating rectangular ducts, J. Heat Transfer 107 (3) (1985) 575-582.

[5] E. Levy, S. Neti, G. Brown, F. Bayat, V. Kadambi, Laminar heat transfer and pressure drop in a rectangular duct rotating about a parallel axis, J. Heat Transfer 108 (2) (1986) 350-356.

[6] C.Y. Soong, W.M. Yan, Development of secondary flow and convective heat transfer in isothermal/iso-flux rectangular ducts rotating about a parallel axis, Int. J. Heat Mass Transfer 42 (1999) 497-510.

[7] A.K. Sleiti, J.S. Kapat, Heat transfer in channels in parallel-mode rotation at high rotation numbers, Journal of Thermophysics and Heat Transfer 20 (4) (2006) 748-753.

[8] D.A. Nield, A. Bejan, Convection in porous media, fourth ed., Springer, New York, 2013.

[9] L. Tianjian, Ultralight porous metals: from fundamentals to applications, Acta Mech. Sin. Chin. J. Mech. 18 (5) (2002) 457-479.

[10] M.L. Hunt, C.L. Tien, Effects of thermal dispersion on forced convection in fibrous media, Int. J. Heat Mass Transfer 31 (2) (1988) 301-309.

[11] K. Boomsma, D. Poulikakos, F. Zwick, Metal foams as compact high performance heat exchangers, J. Mech. Mater. 35 (2003) 1161-1176.

[12] V.V, Calmidi, Transport phenomena in high porosity metal foams, PhD thesis, University of Colorado, 1998.

[13] A. Amiri, K. Vafai, T.M. Kuzay, Effects of boundary conditions on non-darcian heat transfer through porous media and experimental comparisons, Numerical Heat Transfer, Part A (1995) 651-664.

[14] D-Y. Lee, K. Vafai, Analytical characterization and conceptual assessment of solid and fluid temperature differentials in porous media, Int. J. Heat Mass Transfer 42 (1999) 423-435.

[15] B. Alazmi, K. Vafai, Constant wall heat flux boundary conditions in porous media under local thermal non-equilibrium conditions, Int. J. Heat Mass Transfer 45 (2002) 3071-3087.

[16] S. Mahjoob, K. Vafai, Analytical characterization and production of an isothermal surface for biological and electronic applications, J. Heat Transfer 131 (2009) p. 052604.

[17] S. Mahjoob, K. Vafai, Analysis of heat transfer in consecutive variable cross-sectional domains: applications in biological media and thermal management, J. Heat Transfer 133 (2011) p. 011006.

[18] V.V. Calmidi, R.L. Mahajan, The effective thermal conductivity of high porosity fibrous metal foams, J. Heat Transfer 121 (1999) 466-471.

[19] V.V. Calmidi, R.L. Mahajan, Forced convection in high porosity metal foams, J. Heat Transfer 122 (2000) 557-565.

[20] S. Mahjoob, K. Vafai, A synthesis of fluid and thermal transport models for metal foam heat exchangers, Int. J. Heat Mass Transfer 51 (2008) 3701-3711.

[21] C.Y. Zhao, Review on thermal transport in high porosity cellular metal foams with open cells, Int. J. Heat Mass Transfer 55 (2012) 3618-3632. 
[22] P. Vadasz, Flow in Rtating Porous Media, in: P. du Plessis (Ed.), Fluid transport in porous media, in: M. Rahman (Ed.), Advances in fluid mechanics, Vol. 13, Computational Mechanics Publications, Southampton, 1997, pp. 161-214.

[23] P. Vadasz, Fluid flow through heterogeneous porous media in a rotating square channel, Transport in Porous Media 12 (1993) 43-54.

[24] M.A. Havstad, P. Vadasz, Numerical solution of the three-dimensional fluid flow in a rotating heterogeneous porous channel, Int. J. Numerical Methods in Fluids 31(1999) 411-429.

[25] P. Vadasz, Centrifugally generated free convection in a rotating porous box, Int. J. Heat Mass Transfer 37 (13) (1994) 2399-2404.

[26] P. Vadasz, Stability of free convection in a rotating porous layer distant from the axis of rotation, Transport in Porous Media 23(1996) 153-173.

[27] P. Vadasz, Convection and stability in a rotating porous layer with alternating direction of the centrifugal body force, Int. J. Heat Mass Transfer 39 (8) (1996) 1639-1647.

[28] A. Alhusseny, A. Turan, A numerical study of double-diffusive flow in a long rotating porous channel, Heat Mass Transfer 51 (4) ( 2015) 497-505.

[29] S.C. Tzeng, C.Y. Soong, S.C. Wong, Heat transfer in rotating channel with open cell porous aluminum foam, Int. Commun. Heat Mass Transfer 31 (2) (2004) 261-272.

[30] A. Alhusseny, A. Turan, Effects of centrifugal buoyancy on developing convective laminar flow in a square channel occupied with a high porosity fibrous medium, Int. J. Heat Mass Transfer 82 (2015) 335-347.

[31] A. Alhusseny, A. Turan, A. Nasser, Hydrodynamically and thermally developing flow in a rectangular channel filled with a high porosity fiber and rotating about a parallel axis, In Press: International Communications in Heat Mass and Transfer.

[32] D. Poulikakos, M. Kazmierczak, Forced convection in duct partially filled with a porous material, J. Heat Transfer, 109 (3) (1987) 653-662.

[33] S. Chikh, A. Boumedien, K. Bouhadef, G. Lauriat, Analytical solution of non-darcian forced convection in an annular duct partially filled with a porous medium, Int. J. Heat Mass Transfer, 38 (9) (1995) 1543-1551.

[34] K. Vafai, S. J. Kim, Analysis of Surface Enhancement by a Porous Substrate, J. Heat Transfer, 112 (3) (1990) 700-706.

[35] M. Chandesris, D. Jamet, Boundary conditions at a planar fluid-porous interface for a poiseuille flow, Int. J. Heat Mass Transfer, 49 (2006) , 2137-2150.

[36] J.A. Ochoa-Tapia, S. Whitaker, Heat transfer at the boundary between a porous medium and a homogeneous fluid, Int. J. Heat Mass Transfer 40 (11) (1997) 2691-2707.

[37] H.J. Xu, Z.G. Qu, T.J. Lu, Y.L. He, W.Q. Tao, Thermal modeling of forced convection in a parallel-plate channel partially filled with metallic foams, J. Heat Transfer 133 (2011) p. 092603.

[38] S.V. Patankar, D.B. Spalding, A calculation procedure for heat, mass and momentum transfer in three-dimensional parabolic flows, Int. J. Heat Mass Transfer 15 (1972) 1787-1806.

[39] D.B. Spalding, A novel finite-difference formulation for differential expressions involving both first and second derivatives, Int. J. Numer. Methods Eng. 4 (1972) 551-559.

[40] K.A. Hoffmann and S.T. Chiang, Computational Fluid Dynamics, 4th ed., Vol. 1, Engineering Education Science 1, USA (2000), pp. 369.

[41] P.M. Kamath, C. Balaji, S.P. Venkateshan, Experimental investigation of flow assisted mixed convection in high porosity foams in vertical channels, Int. J. Heat Mass Transfer 54 (2011) 5231-5241. 

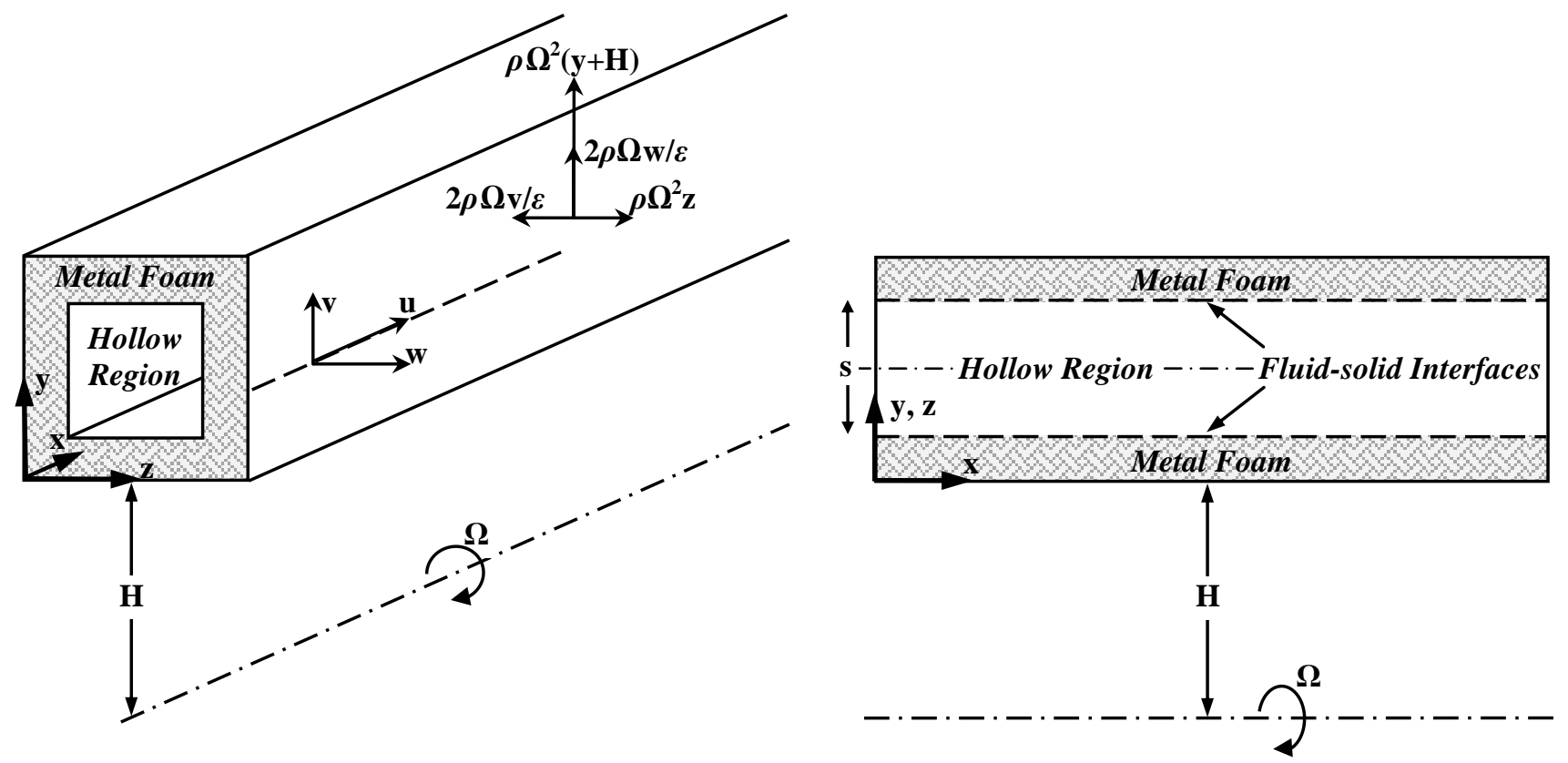

Fig.(1) The geometrical shape of the studied problem 


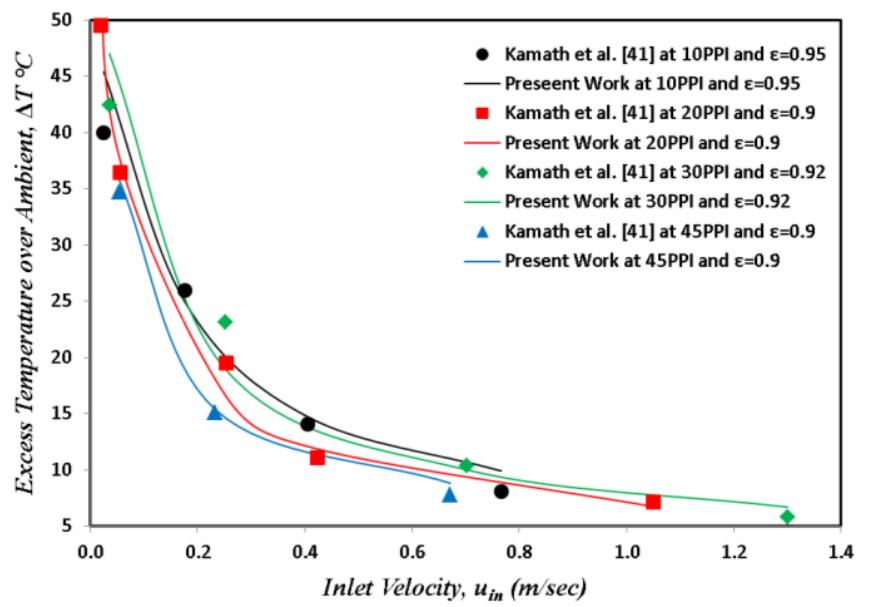

(a)

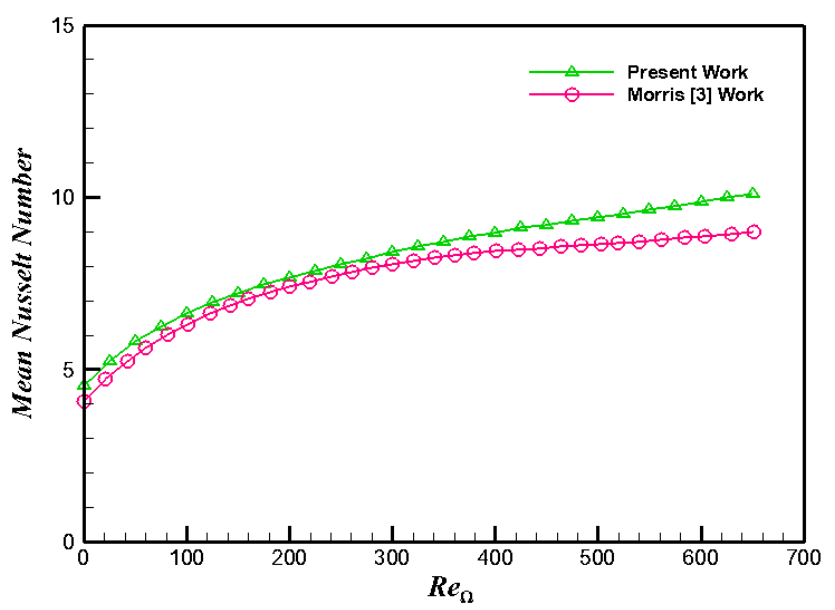

(b)

Fig.(2) Comparing the numerical data obtained with former experiments for a) excess temperature over ambient at $\operatorname{Pr}=0.7$, b) Nusselt number at $\operatorname{Re}=2500, \operatorname{Pr}=0.7$ and $\mathrm{H} / \mathrm{Dh}=32$ 

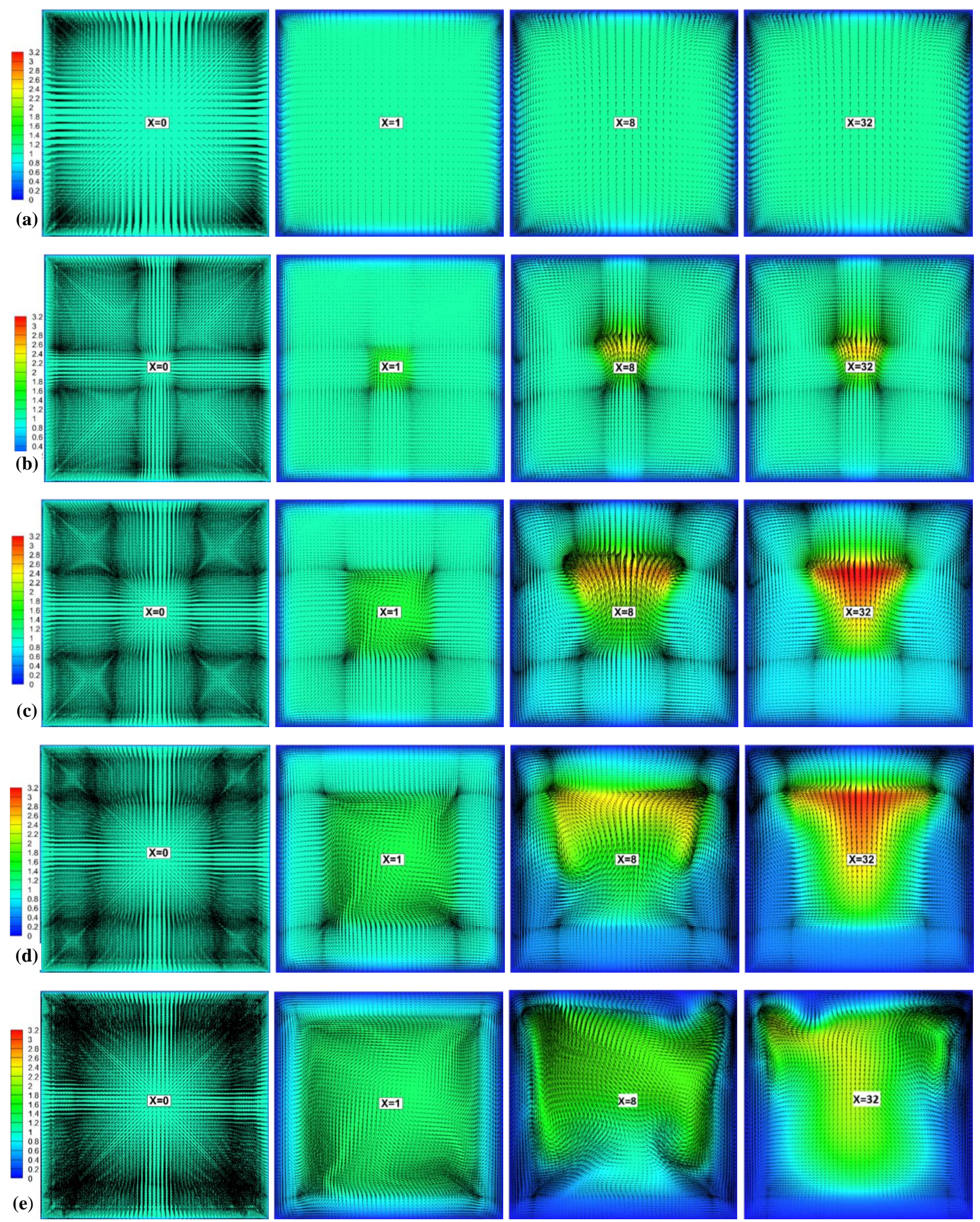

Fig.(3) Axial development of the dimensionless longitudinal velocity and cross-sectional velocity vectors for different hollow ratios; a) $S=0$, b) $S=0.2$, c) $S=0.4$, d) $S=0.6$, e) $S=0.8$ 


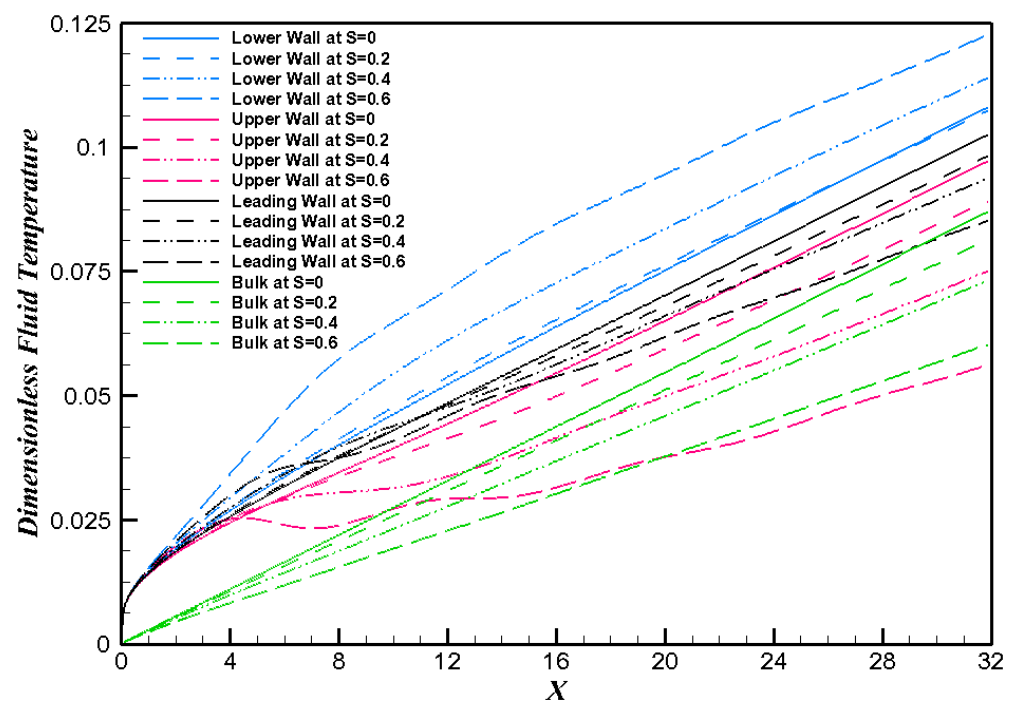

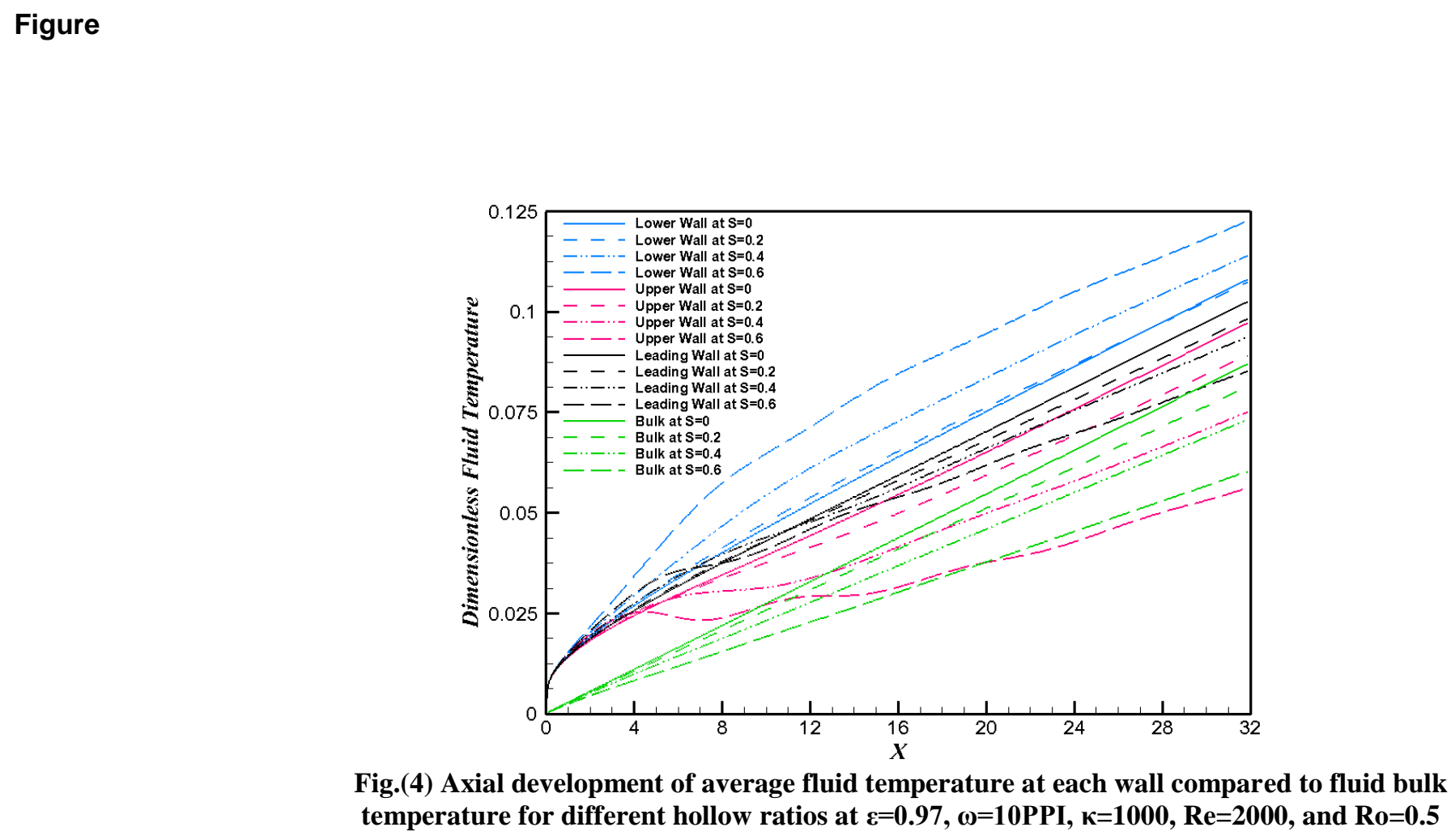

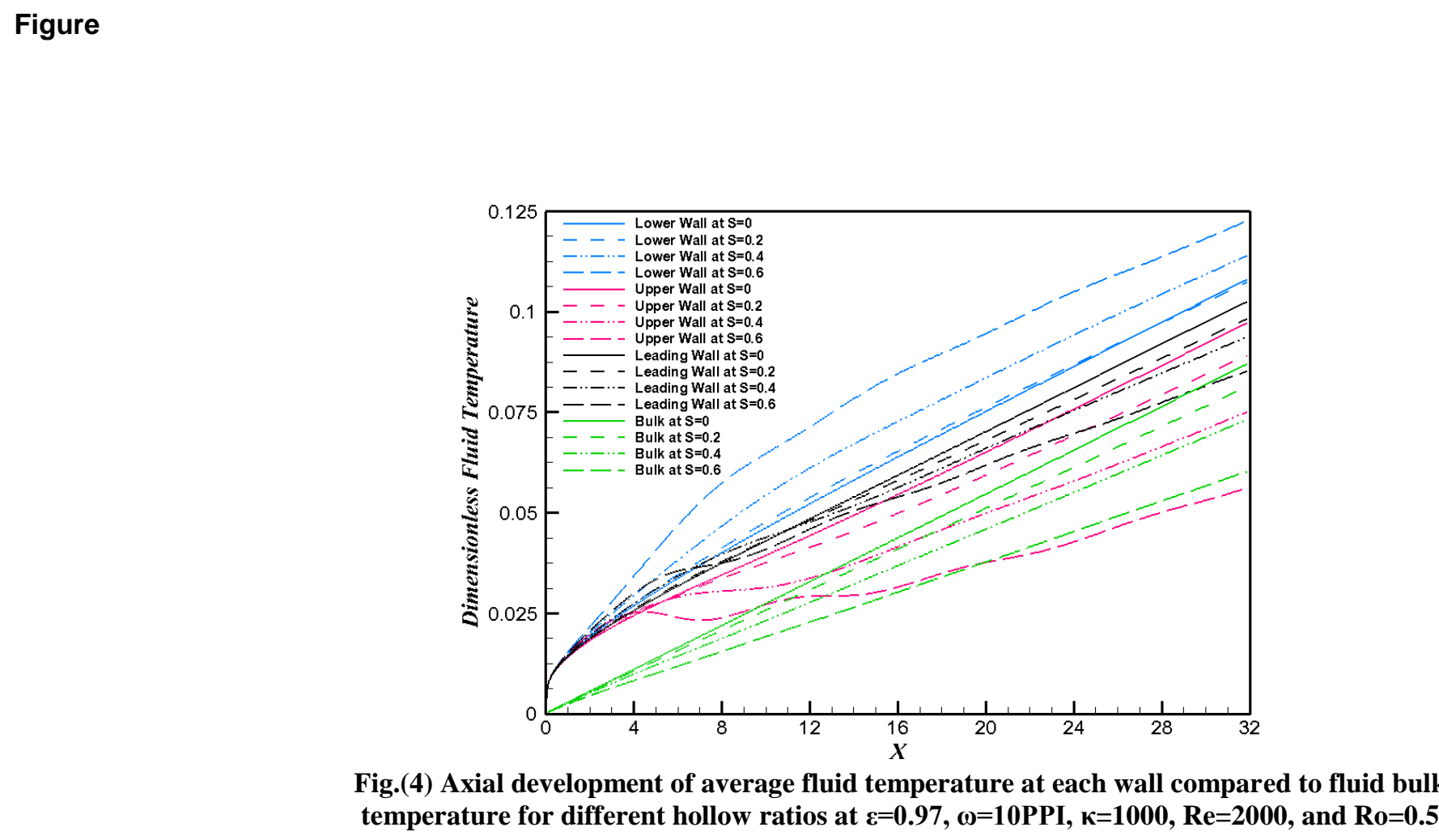

.
(

.
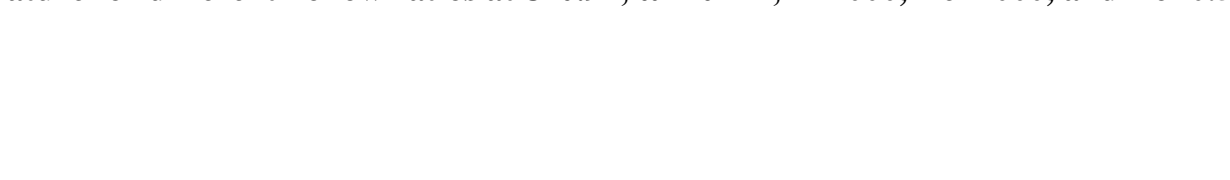


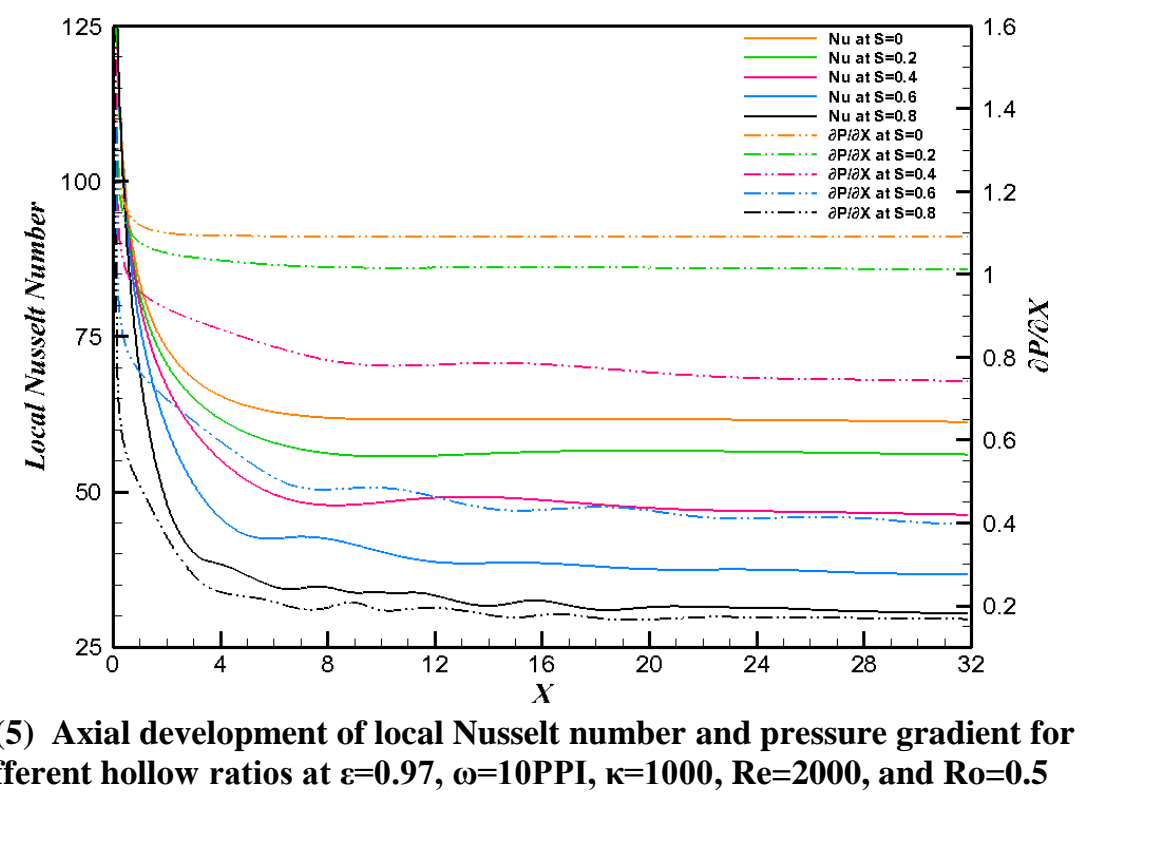

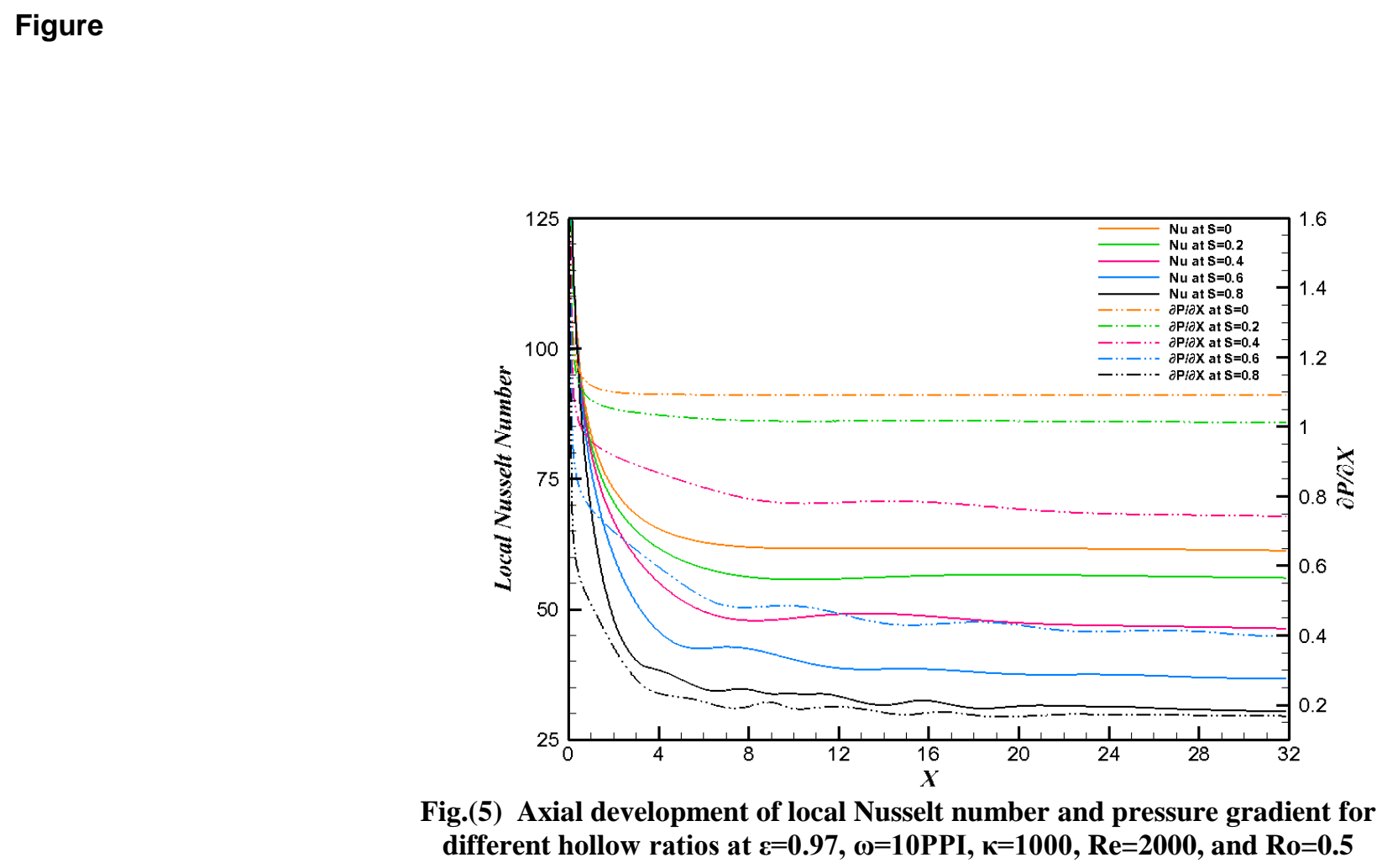

Figure

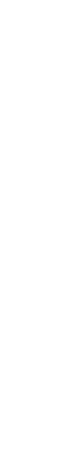




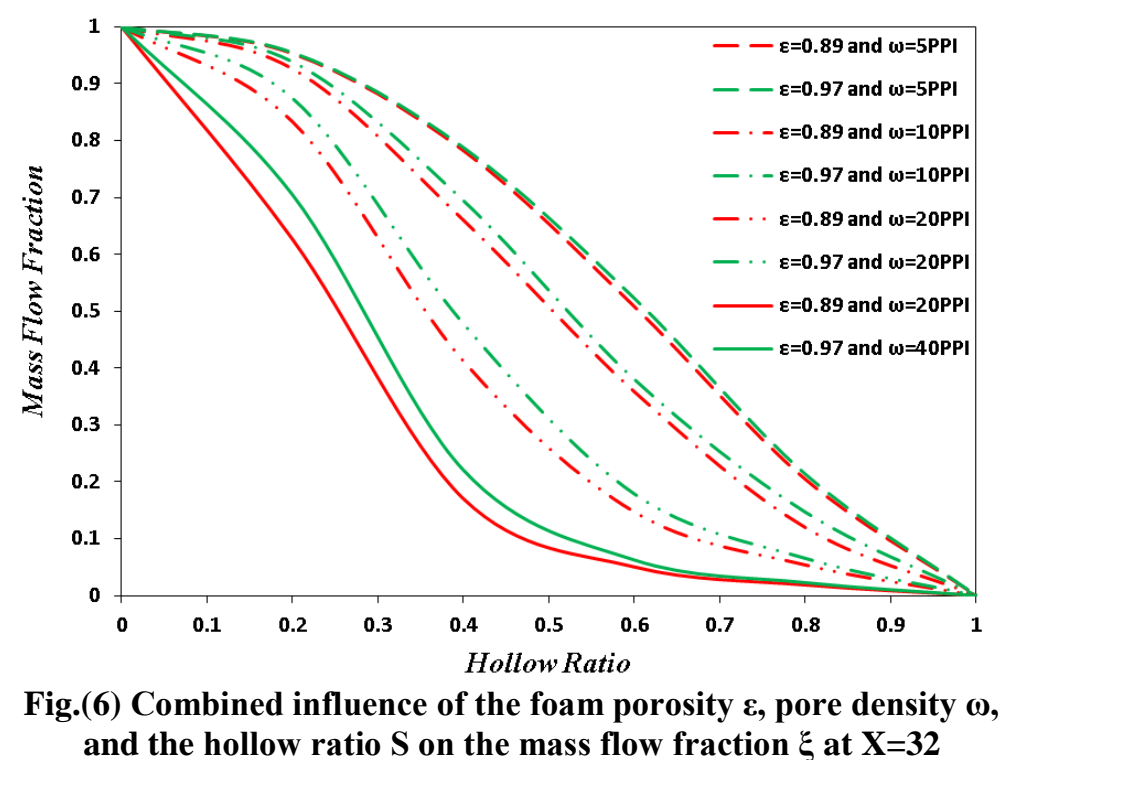

Figure

Figure ,

\begin{abstract}
and the hollow ratio $S$ on the mass flow fraction $\xi$ at $X=32$
\end{abstract}

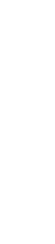

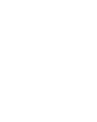
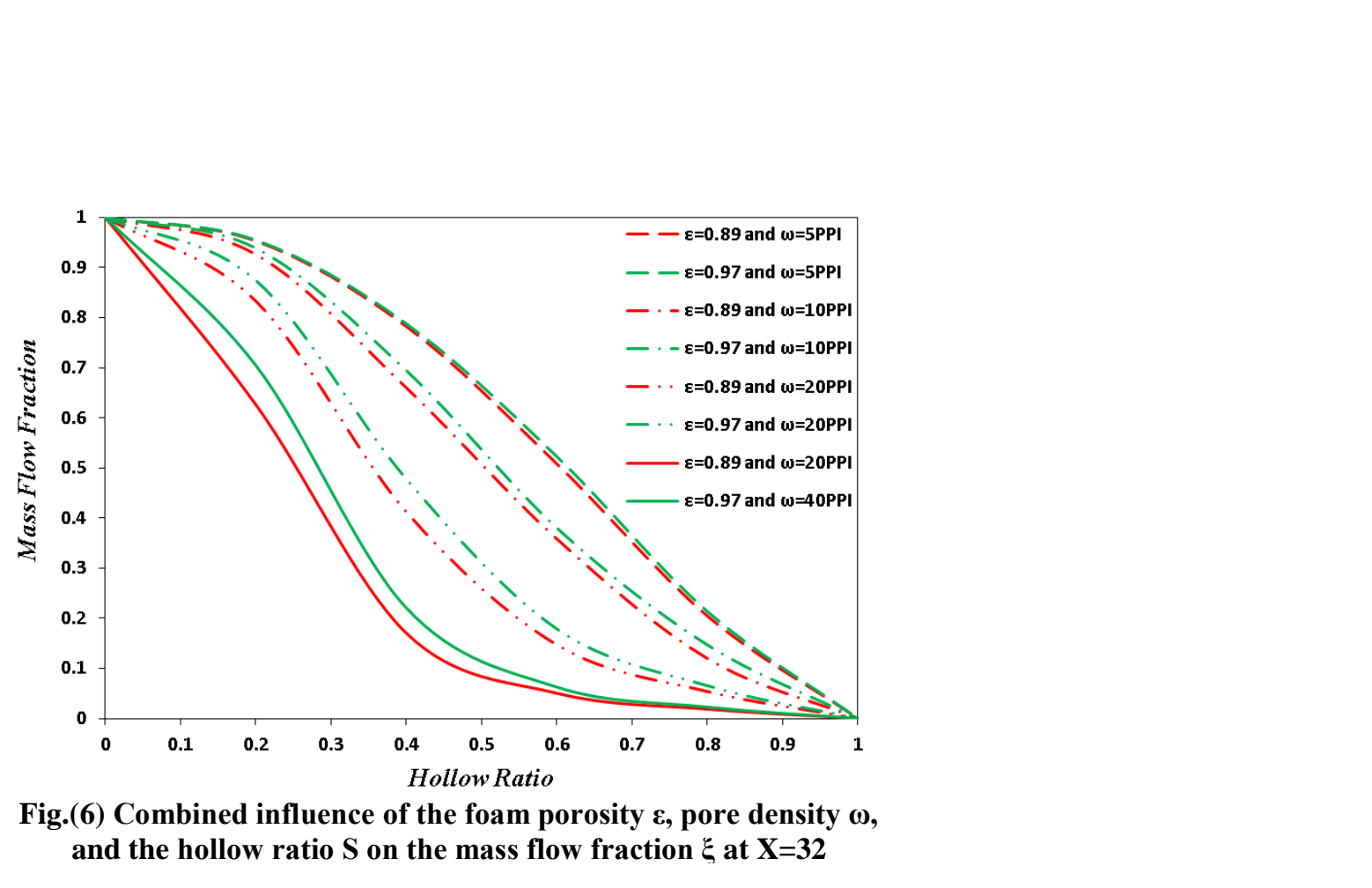

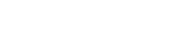

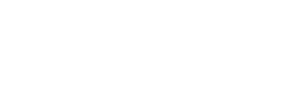




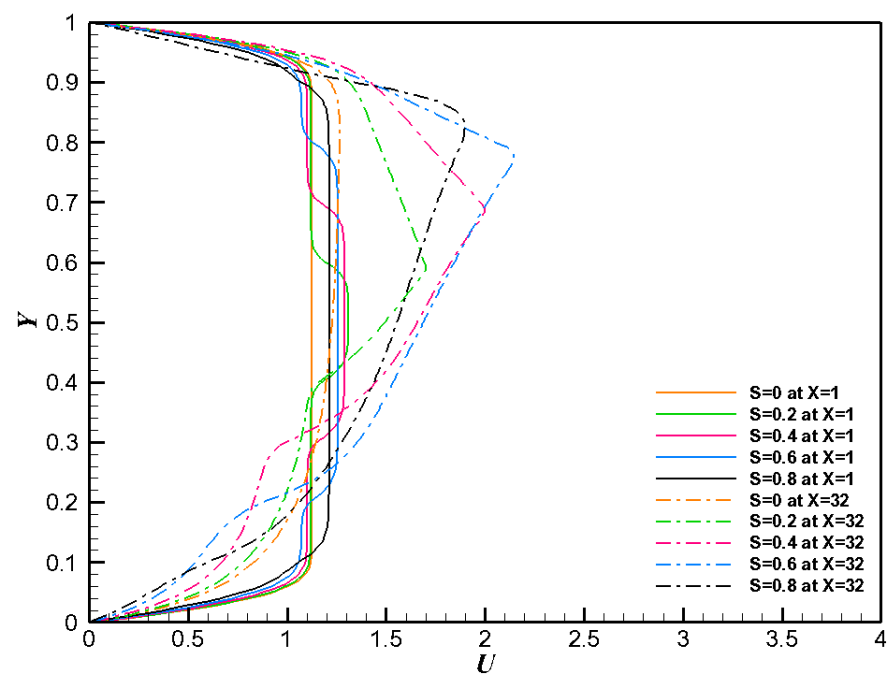

(a)

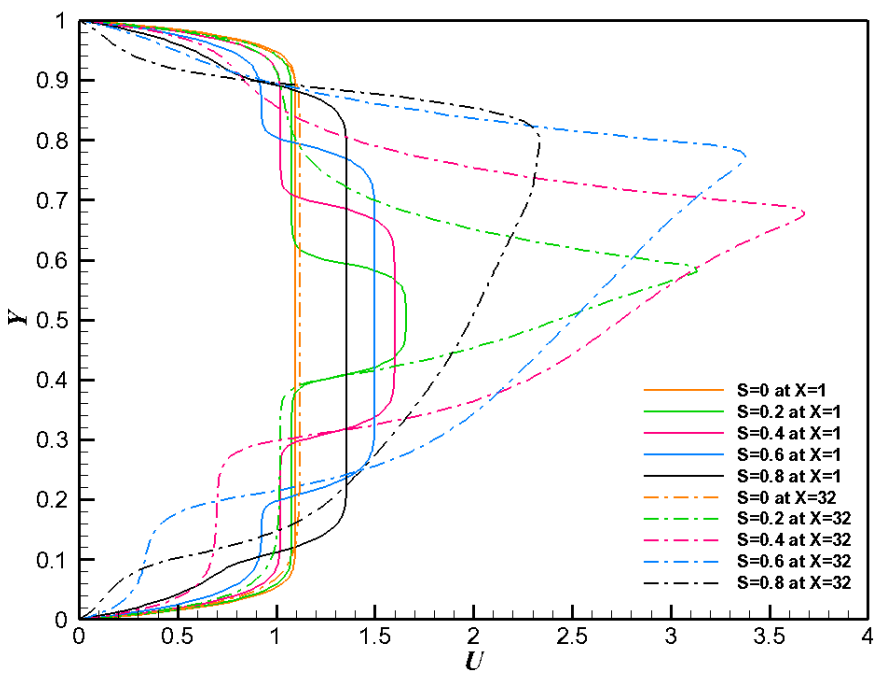

(b)

Fig. (7) Combined effect of pore density and hollow ratio on the development of longitudinal velocity profiles at $\mathrm{Z}=0.5, \varepsilon=0.89$, $\kappa=1000, \mathrm{Re}=2000$, and $\mathrm{Ro}=0.5$; a) $\omega=5 \mathrm{PPI}, \mathrm{b}) \omega=10 \mathrm{PPI}$ 


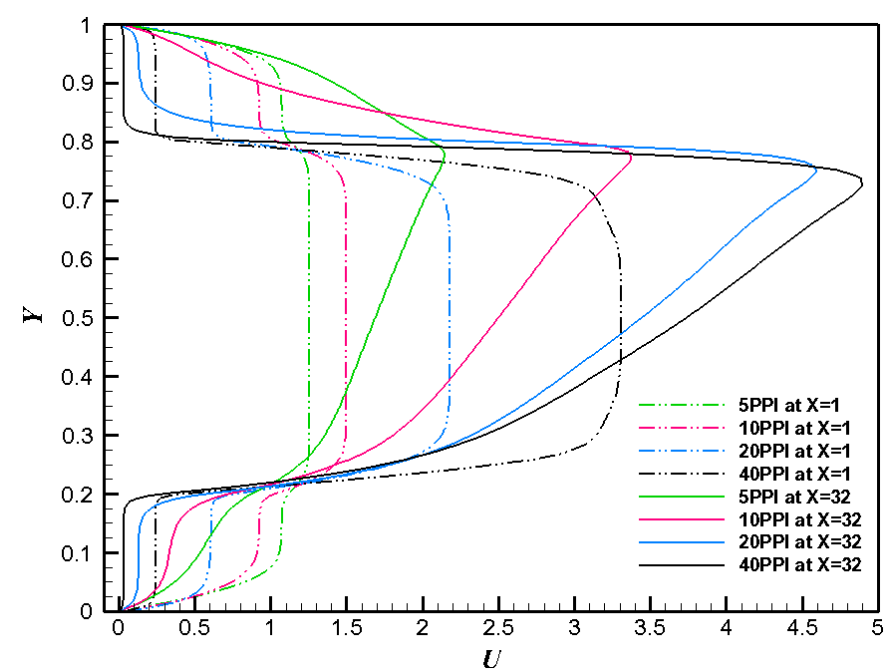

(a)

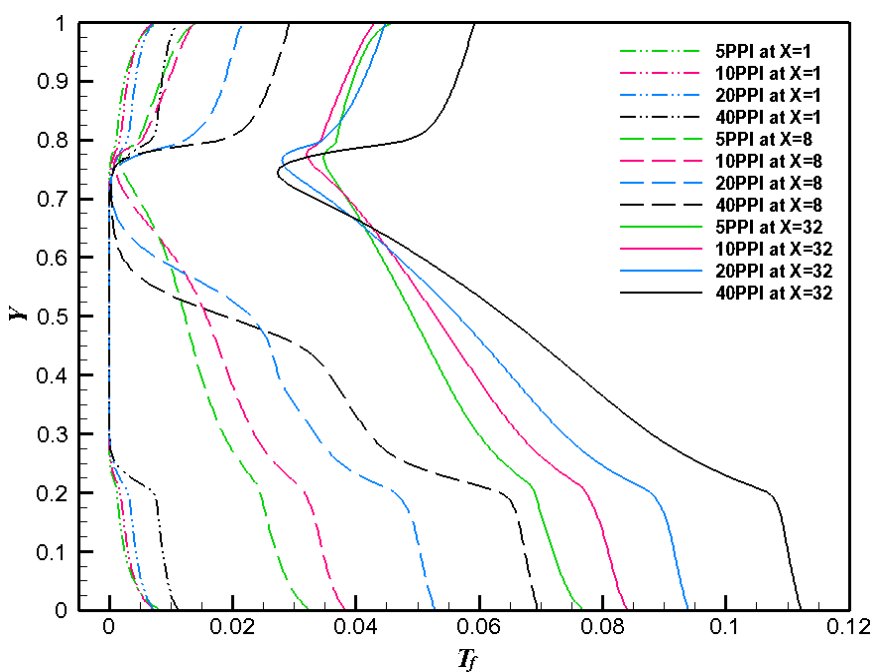

(b)

Fig. (8) Effect of pore density on the development of; a) longitudinal velocity profiles, b) Fluid temperature profiles at $\mathrm{Z}=0.5, \mathrm{~S}=0.6, \varepsilon=0.89, \kappa=1000, \operatorname{Re}=2000$, and $\mathrm{Ro}=0.5$ 


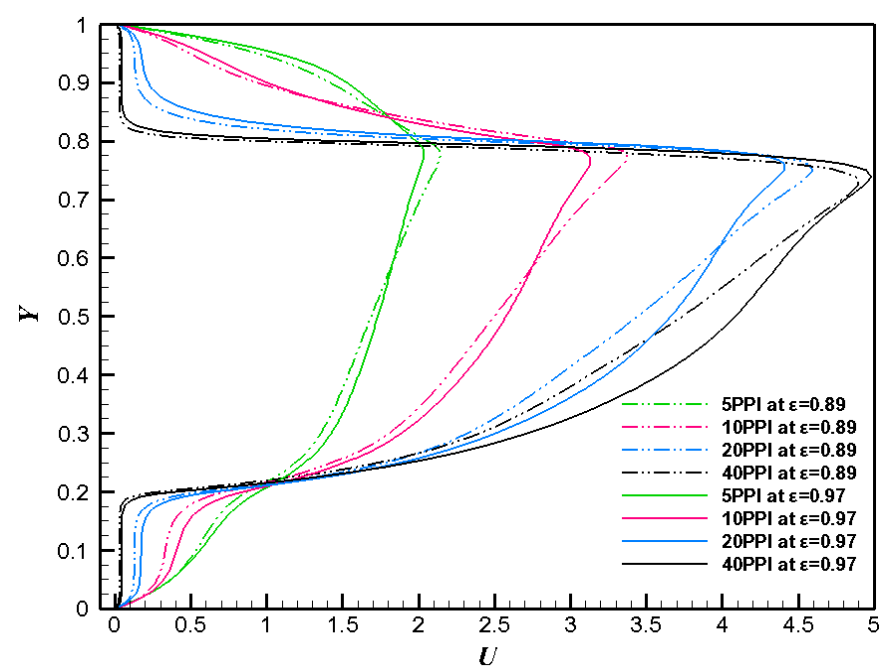

(a)

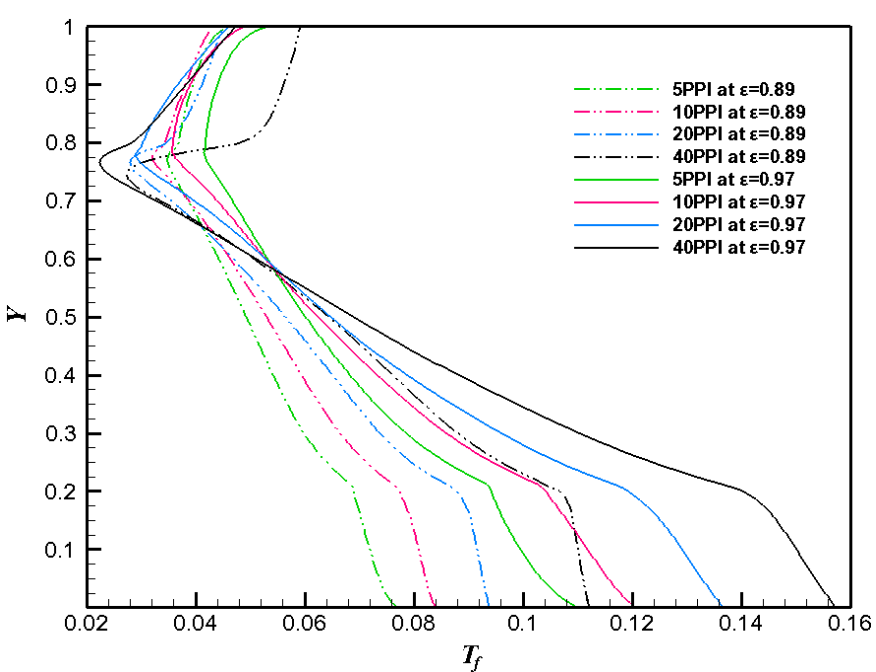

(b)

Fig. (9) Effect of pore density on the development of; a) longitudinal velocity profiles, b) Fluid temperature profiles at $\mathrm{X}=32, \mathrm{Z}=\mathbf{0 . 5}, \mathrm{S}=\mathbf{0 . 6}, \kappa=1000, \mathrm{Re}=2000$, and $\mathrm{Ro}=0.5$ 


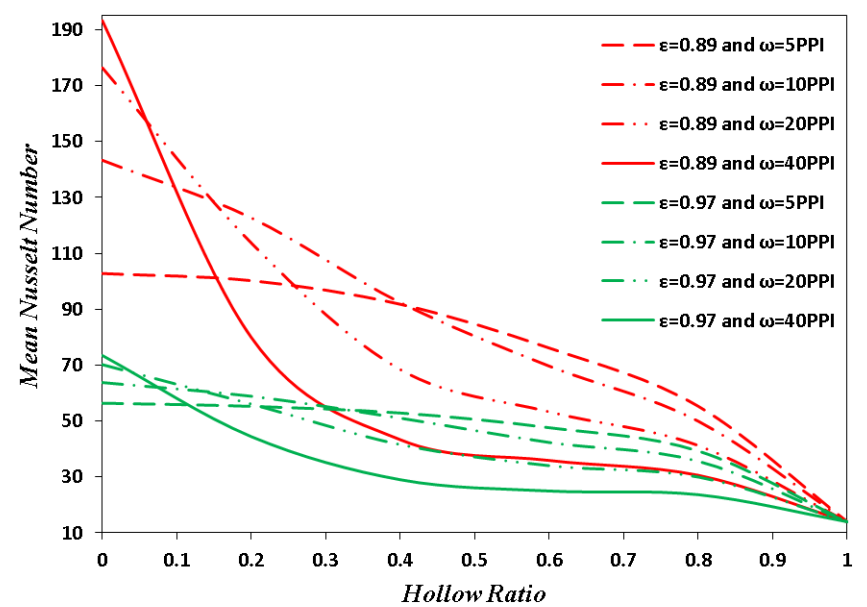

(a)

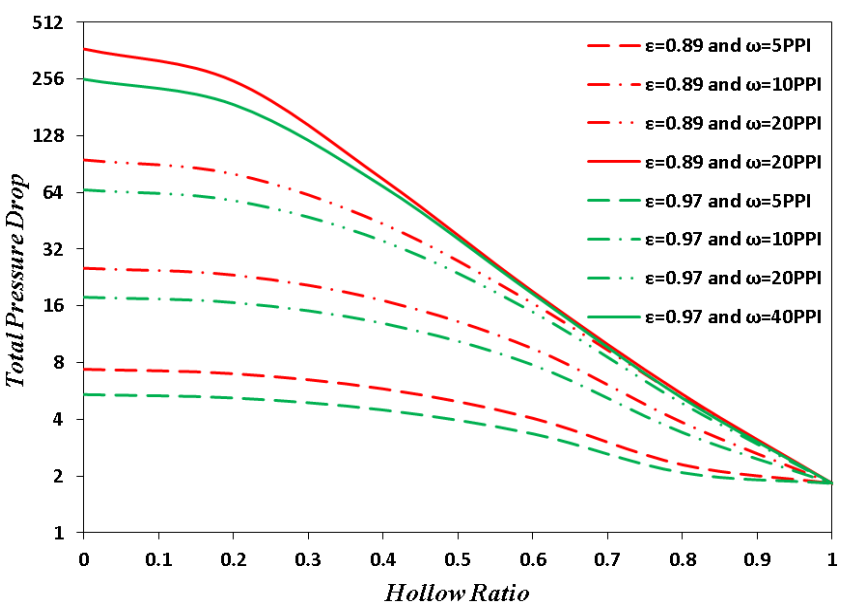

(b)

Fig. (10) Effects of hollow ratio, porosity and, pore density on; a) mean Nusselt number, b) Total pressure drop at $\kappa=1000, \operatorname{Re}=2000$, and $\mathrm{Ro}=0.5$ 

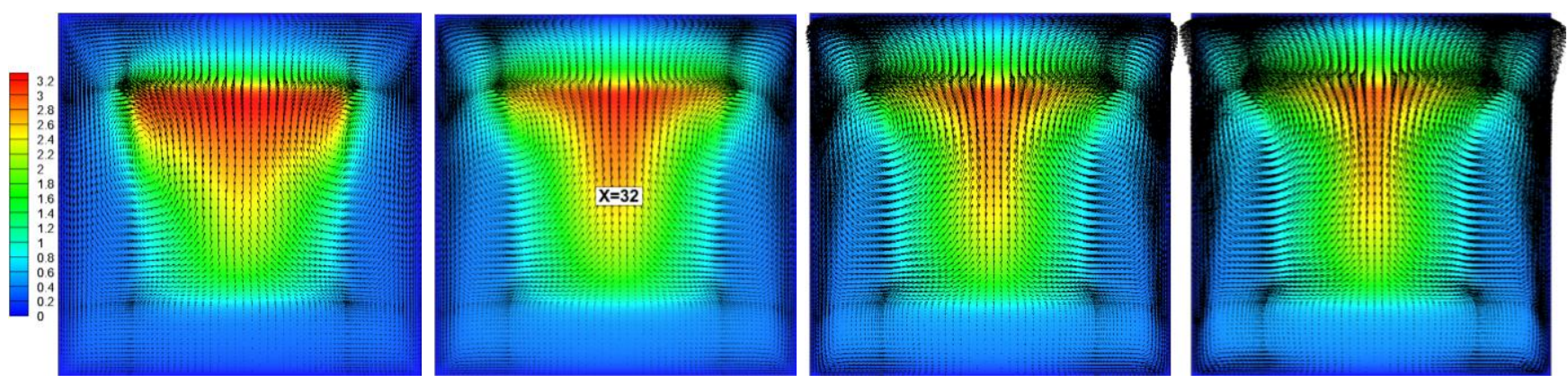

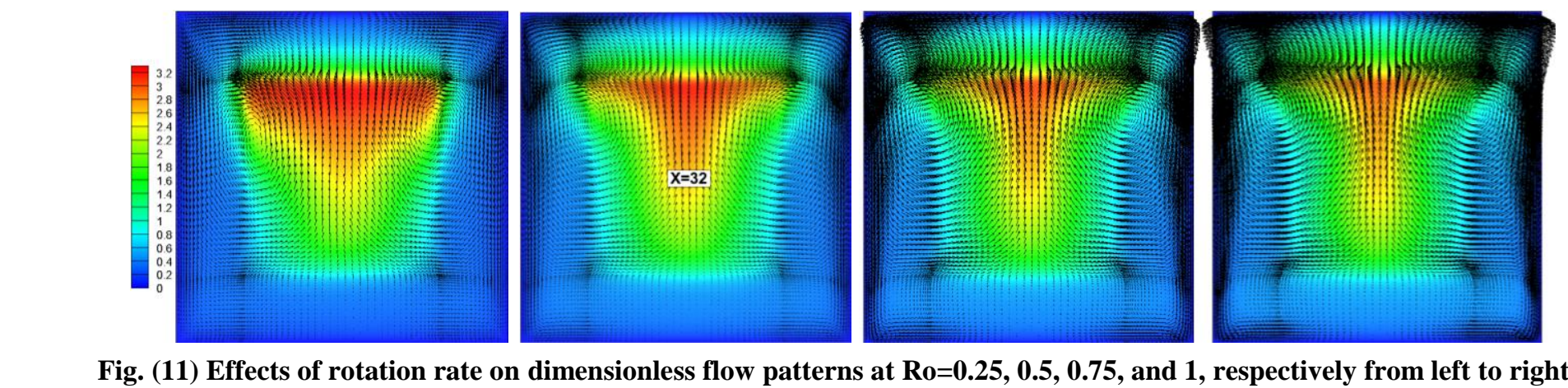

Fig. (11) Effects of rotation rate on dimensionless flow patterns at Ro=0.25, $0.5,0.75$, and 1 respectively from left to right

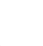
. . . rang

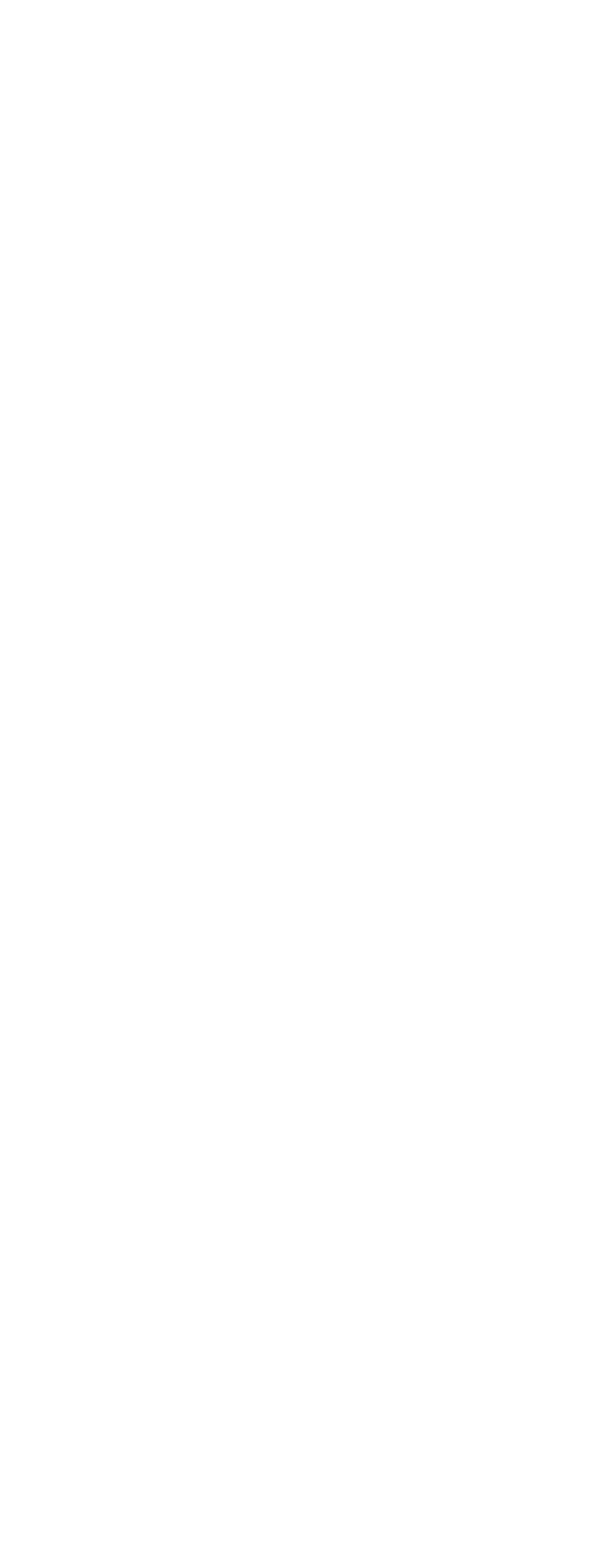




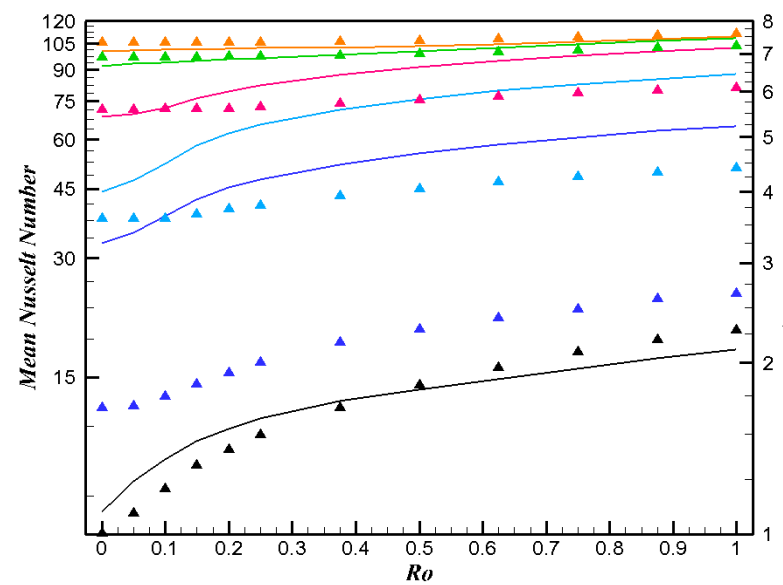

(a)

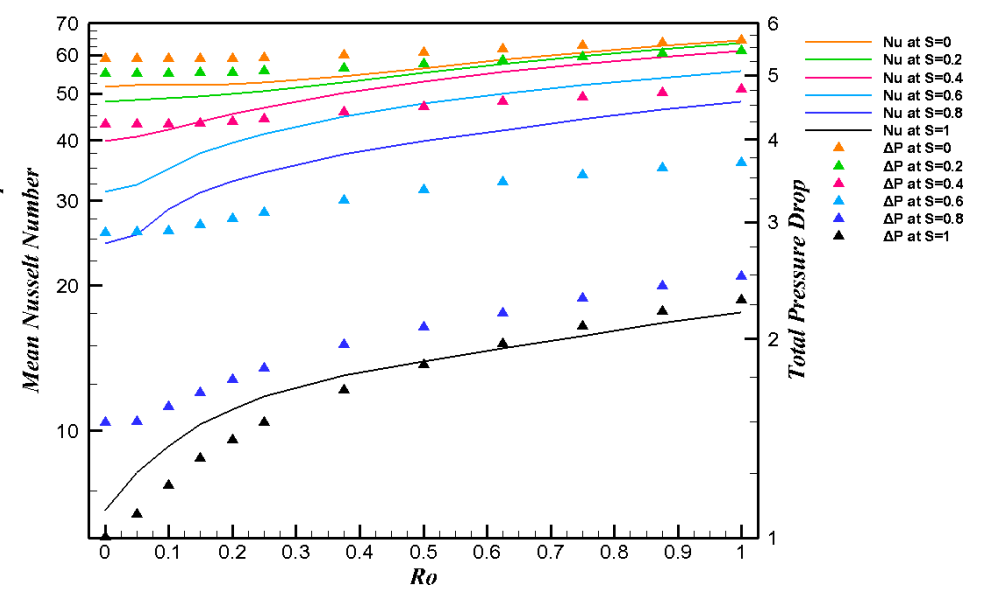

(b)

Fig. (12) Effects of rotation rate and hollow ratio on mean Nusselt number and total pressure drop at $\omega=5 \mathrm{PPI}, \kappa=1000, \operatorname{Re}=2000$; a) $\varepsilon=0.89$, b) $\varepsilon=0.97$ 


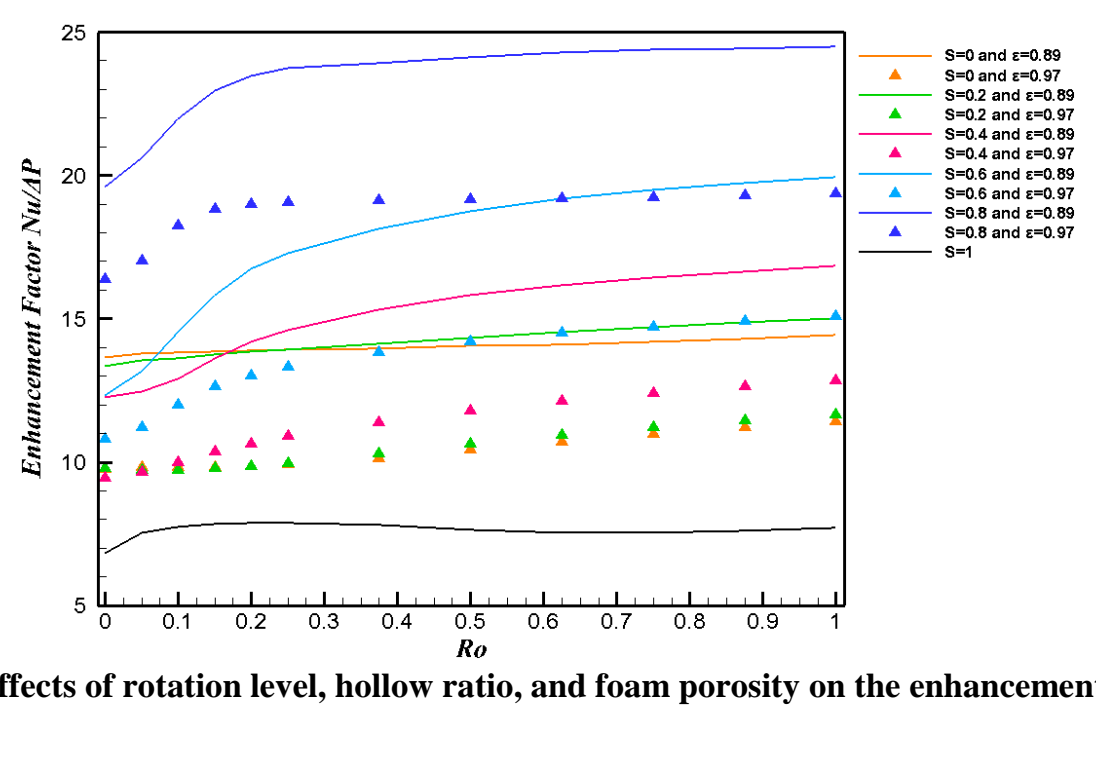

Fig. (13) Effects of rotation level, hollow ratio, and foam porosity on the enhancement factor

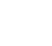

\section{Figure}

Fig. (13) Effects of rotation level, hollow ratio, and foam porosity on the enhancement factor 


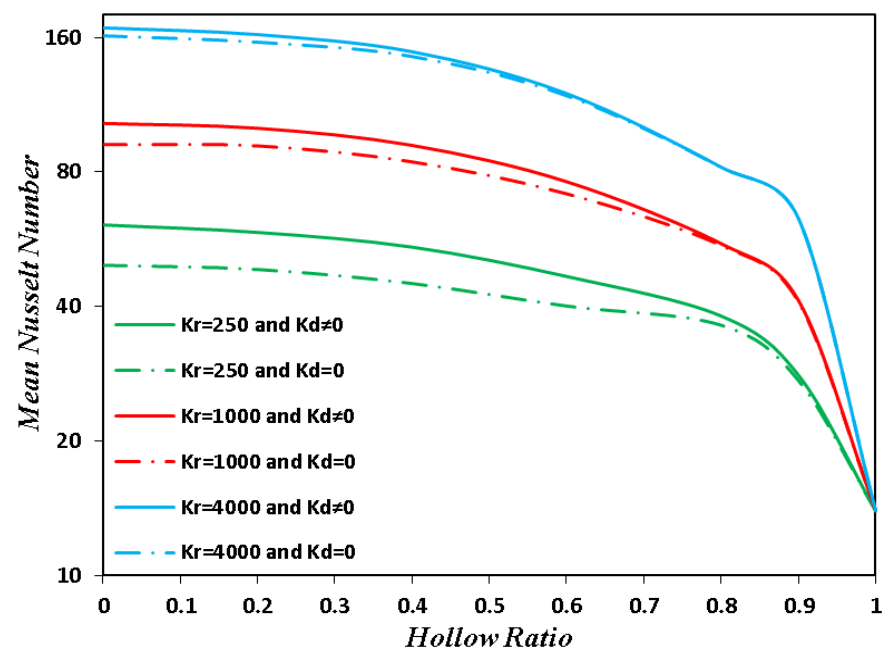

(a)

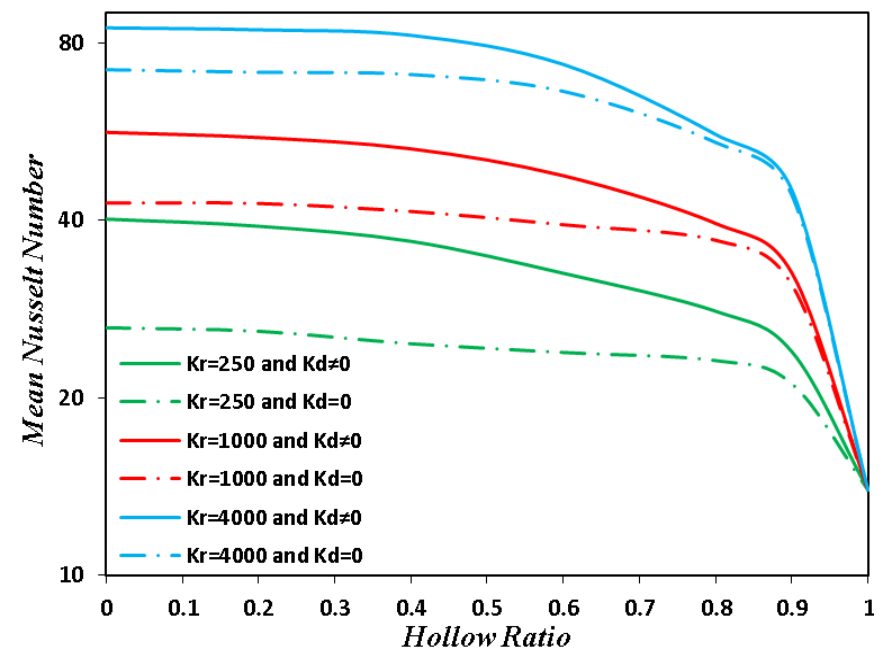

(b)

Fig. (14) The variation of mean Nusselt number with hollow ratio at $\omega=5 P P I, R o=0.5$, and $\operatorname{Re}=2000$ for various values of medium porosity a) $\varepsilon=0.89$, b) $\varepsilon=0.97$ 

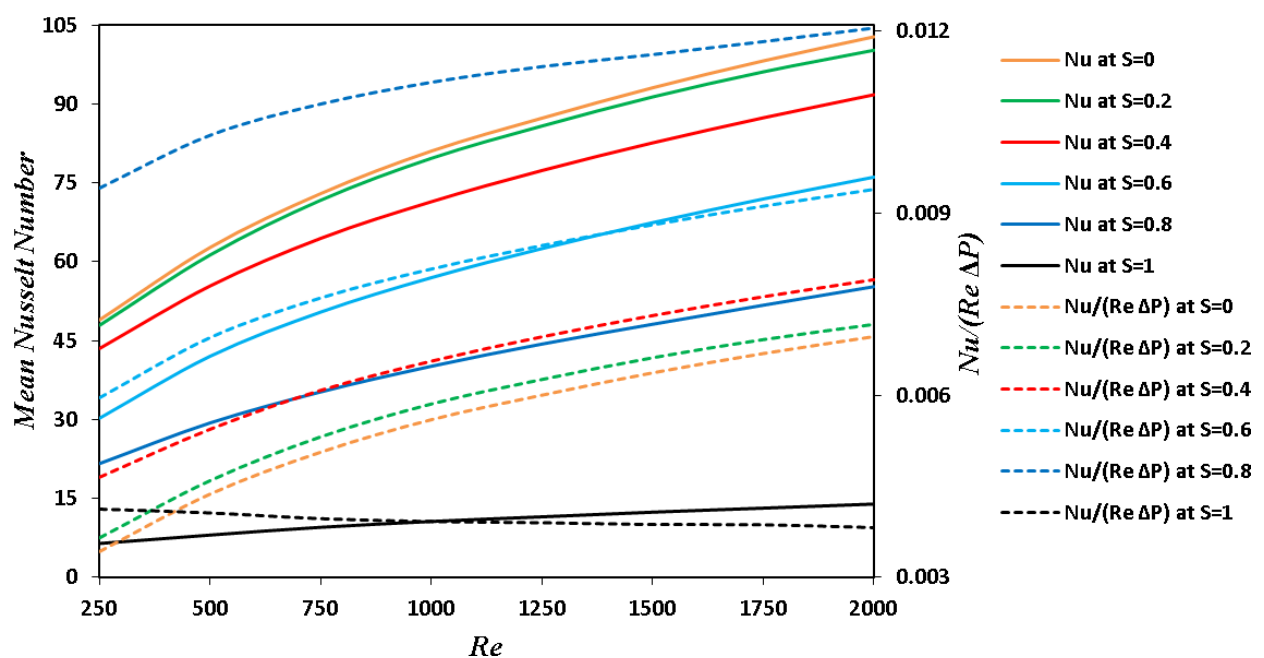

(a)

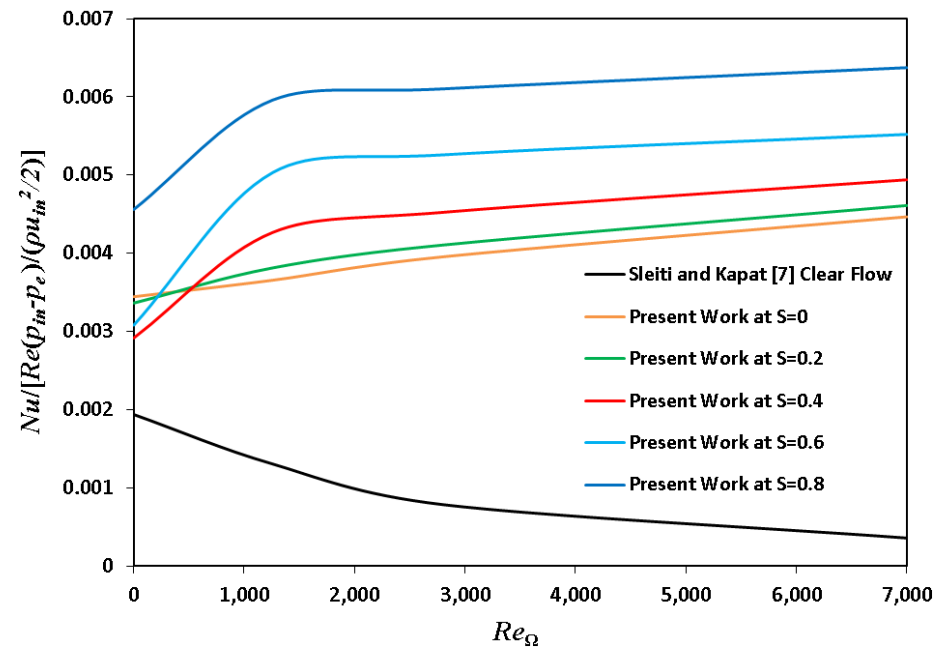

(b)

Fig.(15) The overall enhancement factor $\varepsilon=0.89, \omega=5 P P I, \kappa=1000$; a) The impact of flow Reynolds number at $\mathrm{Ro}=\mathbf{0 . 5}$, b) Comparison with a clear turbulent flow 
We certify that the present manuscript has not been published in an archival journal. Also, we certify that the present manuscript is not currently submitted for publication in another journal.

Authors 